\title{
The RNF26/CBX7 axis modulates the TNF pathway to promote cell proliferation and regulate sensitivity to TKIs in ccRCC
}

\author{
Wentao Liu1 ${ }^{1,2,3^{*}}$, Haohui Wang1,2,3* , Chengzhu Jian ${ }^{1,2^{*}}$, Wei Li1,2, Kun Ye², Jiannan Ren ${ }^{1,2}$, Liang Zhu ${ }^{1,2}$, \\ Yinhuai Wang ${ }^{1}$, Xin Jin ${ }^{1,2}{ }^{凶}$, Lu Y $i^{1,2,3 凶}$ \\ 1. Department of Urology, The Second Xiangya Hospital, Central South University, Changsha, Hunan, 410011, China \\ 2. Uro-Oncology Institute of Central South University, Changsha, Hunan, 410011, China \\ 3. Hunan Engineering Research Center of Smart and Precise Medicine, Changsha, Hunan, 410011, China \\ *These authors contributed equally. \\ $\triangle$ Corresponding authors: Xin Jin (E-mail: jinxinxy2@csu.edu.cn; Tel: +87 17786430489; Fax number: 0731-85295888; Mail address: No. 139, Renmin Road \\ Central, Changsha, Hunan, China, 410011); Lu Yi (E-mail: yilu9999@csu.edu.cn; Tel: +87 15364090896; Fax number: 0731-85295888; Mail address: No. 139, \\ Renmin Road Central, Changsha, Hunan, China, 410011).
}

(c) The author(s). This is an open access article distributed under the terms of the Creative Commons Attribution License (https://creativecommons.org/licenses/by/4.0/). See http://ivyspring.com/terms for full terms and conditions.

Received: 2021.11.22; Accepted: 2022.02.09; Published: 2022.02.28

\begin{abstract}
Clear cell renal cell carcinoma (ccRCC) accounts for $85 \%$ of all malignant renal tumors. Currently, the pathogenesis of ccRCC is not fully understood. Chromobox (CBX) family proteins are the major subunits of PcG complexes and are implicated in regulating mammalian development. The CBX family consists of eight members, namely, CBX1-8. Numerous studies have highlighted that each CBX protein exhibits distinct functions and prognostic roles in specific cancer types. In this study, in silico analysis indicated that CBX7 was downregulated in $\mathrm{ccRCC}$ and correlated with favorable prognosis in a ccRCC cohort. Subsequent studies showed that $C B X 7$ inhibited cancer cell proliferation and invasion. Then, we showed that CBX7 downregulated ETS1 to inactivate the tumor necrosis factor (TNF) signaling pathway, which inhibited tumor proliferation and enhanced the sensitivity of ccRCC cells to tyrosine kinase inhibitors (TKIs). Moreover, we found that CBX7 was a bona fide substrate of RNF26. RNF26 promoted the degradation of $C B X 7$ and enhanced $c C R C C$ tumor growth. Therefore, our results revealed a novel RNF26/CBX7 axis that modulates the TNF signaling pathway in ccRCC.
\end{abstract}

Key words: CBX7, RNF26, TNF signaling pathway, clear cell renal cell carcinoma

\section{Introduction}

Renal cell carcinoma (RCC) is a heterogeneous group of cancers derived from the renal epithelium [1]. Clear cell RCC (ccRCC) is the major histological subtype of RCC and exhibits distinct characteristics, including genomic alterations and altered tumor metabolism [2]. Approximately $85 \%$ of all RCC tumors are cCRCC tumors. Since ccRCC does not respond to traditional chemotherapy and radiotherapy [2], exploring novel therapeutic strategies has always been a hot spot in ccRCC research. Over the past two decades, targeted antiangiogenesis therapies, including tyrosine kinase inhibitors (TKIs), have profoundly increased the survival time of patients, due to the abnormal hyperactivation of the vascular endothelial growth factor (VEGF) pathway commonly observed in ccRCC [3], which implies that understanding the pathogenesis of ccRCC forms the basis for attempts to identify novel treatment options [4].

Polycomb group (PcG) complexes act as epigenetic regulators to repress target gene expression in cells [5]. Chromobox (CBX) family proteins are the major subunits of PcG complexes and are implicated in regulating mammalian development [6]. The CBX family consists of eight members, namely, CBX1-8. Numerous studies have highlighted that each $C B X$ 
protein exhibits distinct functions and prognostic roles in specific cancer types [7]. It has been mentioned that CBX4 is upregulated in cCRCC and associated with its unfavorable prognosis [8]. Further exploration showed that CBX4 forms a complex with histone deacetylase 1 (HDAC1) to repress the expression of Kruppel like factor 6 (KLF6), which promotes cell growth and migration in ccRCC [8]. However, the specific role of the remaining $\mathrm{CBX}$ family members in ccRCC remains elusive.

In this study, in silico analysis indicated that CBX7 was downregulated in ccRCC and correlated with favorable prognosis in a ccRCC cohort. Subsequent studies showed that CBX7 inhibited cancer cell proliferation and invasion. Then, RNA-seq data for CBX7 indicated that CBX7 inactivated the TNF pathway to block tumor growth and downregulated IL6 to sensitize renal cancer cells to TKIs. Moreover, we found that RNF26 functioned as an E3 ligase for CBX7 and promoted CBX7 degradation in renal cancer cells. Therefore, we identified an RNF26/CBX7 axis that modulates the TNF pathway to regulate the malignant biological behavior of ccRCC cells.

\section{Method and Materials}

\section{Cell lines and cell culture}

The two ccRCC cell lines that we cultured, 786-O and A498, were obtained from Yuchi Biology (Shanghai, China), and both cell lines were identified by short tandem repeat (STR) profiling. Cells were cultured in RPMI-1640 medium (Gibco, USA) or minimum essential medium (MEM, Gibco, USA) supplemented with $10 \%$ fetal bovine serum (FBS; AC03L055, Shanghai Life-iLab Biotech, China) and 1\% penicillin, placed in an incubator at $37^{\circ} \mathrm{C}$ in $5 \% \mathrm{CO} 2$, and the culture medium was changed twice a week.

\section{Antibodies and chemical agents}

The antibodies used for western blot analysis are as follows: GAPDH (\#60004-1-Ig, Proteintech, 1:5000 dilution), RNF26 (\#16802-1-AP, Proteintech, 1:800 dilution), IL6 (\#21865-1-AP, Proteintech, 1:100 dilution), ETS1 (\#12118-1-AP, Proteintech, 1:1000 dilution) and CBX7 (\#26278-1-AP, Proteintech, 1:1000 dilution). NeutraKine ${ }^{\circledR}$ IL6 Monoclonal antibody (Cat no: $69001-1-\mathrm{Ig}) \quad 100 \mathrm{ng} / \mathrm{ml}$ was obtained from Proteintech. MG132 (\#S2619), IKK-16 (\#S2882), JSH-23 (\#S7351) and cycloheximide (CHX) (\#S7418) were purchased from Selleckchem.

\section{Coimmunoprecipitation and western blot analysis}

For coimmunoprecipitation (IP), $1 \mathrm{ml}$ of RIPA buffer (\#P0013, Beyotime, China) containing a protease inhibitor was added to a $10 \mathrm{~cm}$ dish of ccRCC cells for lysis on ice for $30 \mathrm{~min}$. Then, the cell lysates were pipetted into a $1.5 \mathrm{ml}$ Eppendorf tube and centrifuged at $12000 \times \mathrm{g}$ for $15 \mathrm{~min}$. The supernatant was aspirated into a new $1.5 \mathrm{ml}$ Eppendorf tube and incubated with protein A+G beads (\#P2029, Beyotime, China) and IgG (\#A7007, Beyotime, China) or a primary antibody at an ambient temperature of 4 ${ }^{\circ} \mathrm{C}$ with rotational shaking overnight. The next day, the beads were washed 6 times with RIPA buffer, and $60 \mu \mathrm{l}$ of $1 \mathrm{X}$ loading buffer was added. Finally, the beads were boiled in hot water at $100{ }^{\circ} \mathrm{C}$ for 10 minutes. For western blotting, RIPA buffer containing protease inhibitor was added to the cells and placed on ice for $30 \mathrm{~min}$. The lysate was aspirated into Eppendorf tubes and then centrifuged at $12000 \times \mathrm{g}$ for $15 \mathrm{~min}$. The supernatant was removed from the new Eppendorf tubes, and 5X buffer was added. Next, the cell lysate was boiled in hot water $\left(100{ }^{\circ} \mathrm{C}\right)$ for 10 minutes. Finally, the boiled protein lysates were electrophoresed on SDS-PAGE gels. Protein expression levels were measured by using ImageJ software (National Institutes of Health, USA).

\section{Real time RT-PCR analysis}

Total RNA from cells was extracted using TRIzol reagent (\#AG21102, Accurate Biotechnology, Hunan, China). RT-qPCR was performed using reverse transcription kits (\#AG11728, Accurate Biotechnology, Hunan, China) and PCR kits (\#AG11701, Accurate Biotechnology, Hunan, China) according to the manufacturer's instructions. All values were normalized to the corresponding GAPDH values, and the $2^{-\Delta \Delta} \mathrm{Ct}$ method was used to quantify the fold change.

\section{Chromatin immunoprecipitation (ChIP)-qPCR}

The detail of ChIP-qPCR procedure was mentioned previously [9]. The chromatin extraction kit (Abcam, ab117152, USA) and the ChIP Kit Magnetic - One Step (Abcam, ab156907, USA) were employed to performed the ChIP. The primers of genes were provided in the supplementary information.

\section{Cell proliferation assay}

For Cell Counting Kit-8 (CCK-8) assay, CCK-8 reagent (\#C0037, Beyotime) was added to each cell well and an absorbance of $450 \mathrm{~nm}$ was measured with a microplate reader.

All animal experiments were approved by the ethics committee of the Second Xiangya Hospital, Central South University (Approval number: 2021613). BALB/c nude mice (6 weeks old) were purchased from Vital River (Beijing, China). Cells were subcutaneously injected into the left side of the 
backs of the mice $(1 \times 107$ cells per mouse $)$. Tumor volume was calculated using the formula $(\mathrm{L} \times \mathrm{W} 2) / 2$. Once the mice were euthanized, the tumors were excised and weighed.

\section{Tissue microarray and immunohistochemistry (IHC)}

The tissue microarray slides (\# U081ki01) were purchased from bioaitech, China. The tissue microarray specimens were immunostained with RNF26 and CBX7. The method of scoring of staining intensity was mentioned previously [10]. Staining intensity was graded/scored in a blinded fashion: 1 = weak staining at $100 \mathrm{X}$ magnification but little or no staining at $40 \mathrm{X}$ magnification; $2=$ medium staining at 40X magnification; $3=$ strong staining at $40 \mathrm{X}$ magnification [21]. The degree of immunostaining was reviewed and scored by two independent pathologists who were blinded to the clinical details. A final staining index was calculated using the formula: staining intensity $X$ percentage of positive cells.

\section{Statistical analysis}

Data were expressed as means \pm SD. Statistical significance was determined by one-way or two-way ANOVA using GRAPHPAD PRISM 5 software, San Diego, CA, USA. A statistical significance threshold of P-values $<0.05$ was used.

Other methods were provided in the supplementary information.

\section{Results}

\section{CBX7 is a protective factor in renal cell carcinoma}

Since the cancer-related role of the CBX family in renal cell carcinoma is unknown, we first performed bioinformatic analysis and found that CBX7 and CBX6 functioned as protective factors with hazard ratios (HRs) less than 1 but CBX8 and CBX4 functioned as risk factors with HRs greater than 1 in renal cell carcinoma (Fig. 1A). Least absolute shrinkage and selection operator (LASSO)-Cox regression analysis with 1000 replications for these eight CBX family members in the TCGA-KIRC dataset further showed that CBX7 might be a key gene related to overall survival (OS) in renal cell carcinoma (Fig. 1B). Analysis of the TCGA-KIRC dataset also revealed that CBX7 was downregulated in renal cancer tissues compared with nontumor renal tissues (Fig. 1C). This trend was also observed in bladder cancer, prostate cancer and other types of cancer (Supplementary Fig. 1A). Similarly, western blot analysis of the protein levels of CBX7 in patient samples with clear renal cell carcinoma from our hospital indicated that $\mathrm{CBX7}$ was decreased in cancer tissues compared to adjacent nontumor (NAT) renal tissues (Fig. 1D). Moreover, the IHC staining assay of the renal cancer tissue microarray with the anti-CBX7 antibody showed that the protein levels of CBX7 in nontumor tissues were greater than those in renal cancer tissues (Fig. 1E). In addition, we demonstrated that high expression of CBX7 was closely correlated with favorable prognosis in RCC by using the Human Protein Atlas and the GEPIA web tool (Fig. 1F-H). Thus, our data suggest that CBX7 is downregulated in ccRCC and may be a predictor of favorable prognosis in ccRCC.

\section{CBX7 inhibits the progression of renal cancer}

Given that the low expression of CBX7 indicated a worse prognosis of $\mathrm{CCRCC}$, we then investigated the biological role of CBX7 in ccRCC cells. Intriguingly, the single-cell sequence of RCC, acute lymphoblastic leukemia, and ovarian cancer indicated that CBX7 was negatively correlated with angiogenesis, proliferation and invasion (Supplementary Fig. 1B-D). To further test the role of CBX7 in renal cancer cells, CBX7 was knocked down by using gene-specific shRNAs (Fig. 2A, B). CCK-8 and colony formation assays showed that silencing CBX7 enhanced the growth ability of renal cancer cells (Fig. 2C, D). We also demonstrated that knockdown of CBX7 promoted renal cancer cell invasion in vitro (Fig. $2 \mathrm{E}$ ). In contrast, elevation of the CBX7 protein and mRNA levels led to reduced proliferation of renal cancer cells (Fig. 2F-H). Moreover, Tsin-CBX7 was transduced into renal cancer cells to rescue $\mathrm{CBX7}$ expression (Fig. 2I, J). The CCK-8 assay and xenograft assay demonstrated that rescue of CBX7 expression overcame the growth-promoting effect induced by CBX7 downregulation (Fig. 2K-N). Together, these findings briefly show that CBX7 inhibits the aggressive behavior of renal cancer cells.

\section{CBX7 blocks ccRCC tumorigenesis by inhibiting the TNF pathway}

To further explore the mechanism of the tumor suppressive effect of CBX7 in ccRCC, RNA-seq was performed after knockdown of CBX7 (Fig. 3A). KEGG pathway enrichment analysis showed that CBX7 silencing activated the TNF pathway in 786-O cells (Fig. 3B). Gene set enrichment analysis (GSEA) also showed that knockdown of CBX7 was involved in the activation of the TNF signaling pathway (Fig. 3C). In addition, we demonstrated that CBX7 knockdown increased the expression of TNF pathway downstream genes, such as $\mathrm{C}-\mathrm{C}$ motif chemokine ligand 2 (CCL2), interleukin 1 beta (IL1B), C-X-C motif chemokine ligand 2 (CXCL2), TNF, and CXCL3 (Fig. 3D). 
A

$\begin{array}{lrr} & \text { p-value } & \text { HR }(95 \% \text { Cl) } \\ \text { CBX7 } & 5.10 \mathrm{e}-07 & 0.65(0.55 \text { to } 0.77) \\ \text { CBX8 } & 1.33 \mathrm{e}-04 & 1.92(1.37 \text { to } 2.68) \\ \text { CBX6 } & 4.17 \mathrm{e}-03 & 0.76(0.62 \text { to } 0.92) \\ \text { CBX4 } & 1.44 \mathrm{e}-02 & 1.46(1.08 \text { to } 1.97) \\ \text { CBX1 } & 5.18 \mathrm{e}-02 & 0.79(0.63 \text { to } 1.00) \\ \text { CBX5 } & 5.44 \mathrm{e}-02 & 0.81(0.65 \text { to } 1.00) \\ \text { CBX3 } & 6.78 \mathrm{e}-01 & 0.93(0.66 \text { to } 1.31) \\ \text { CBX2 } & 9.91 \mathrm{e}-01 & 1.00(0.76 \text { to } 1.33)\end{array}$

C

Renal cell carcinoma Group 审 Normal 审 Tumor

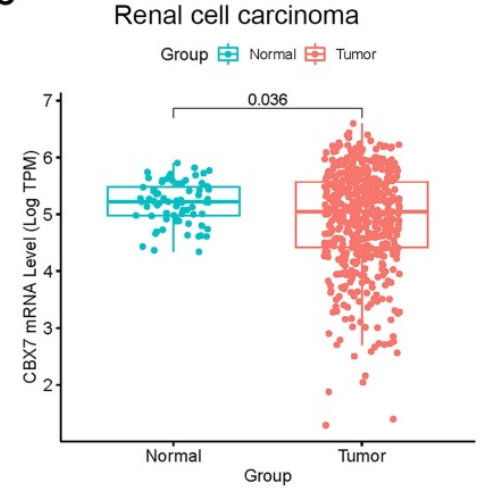

E

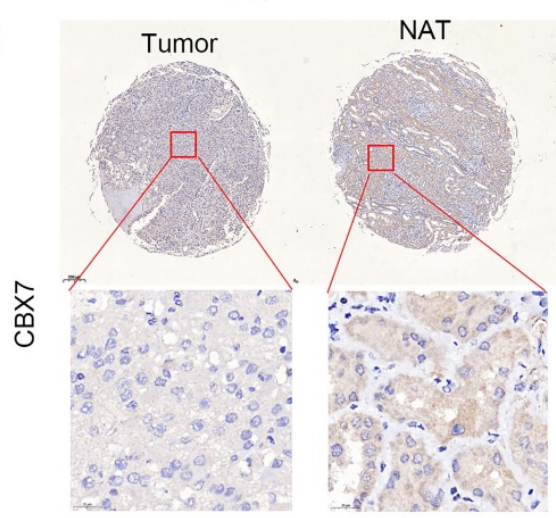

$\mathbf{F}$

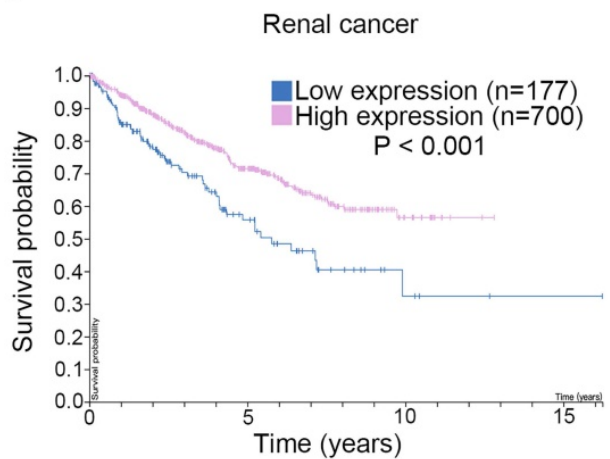

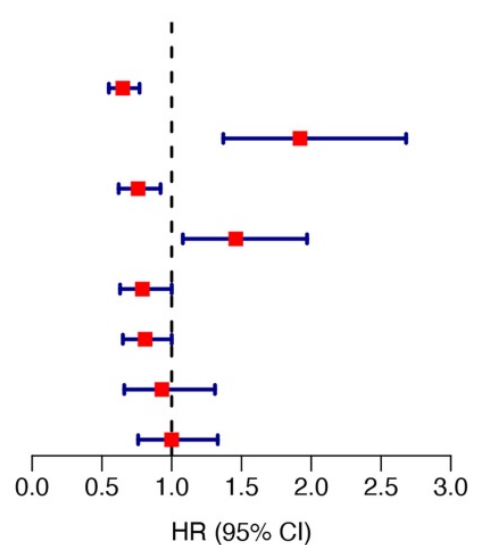

D

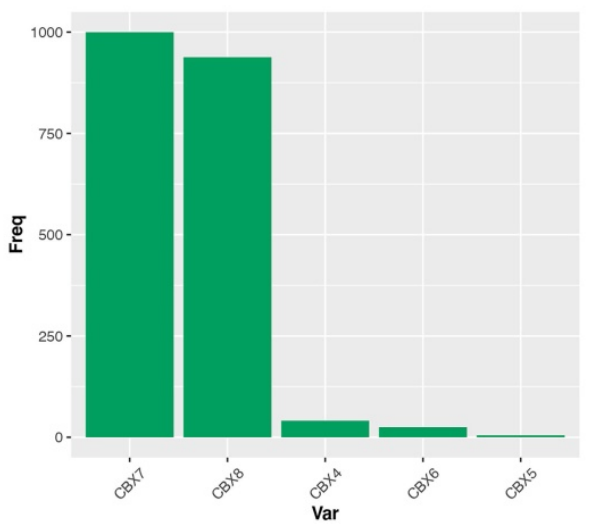

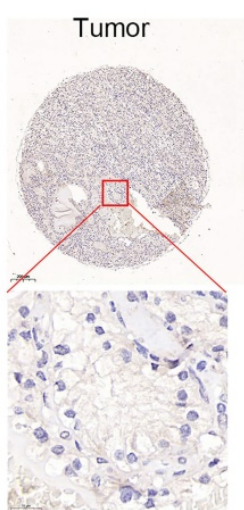

G

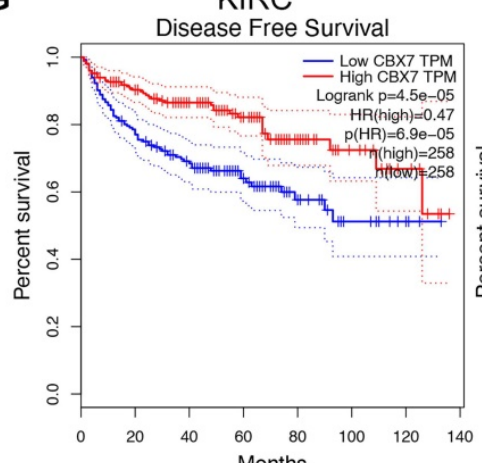

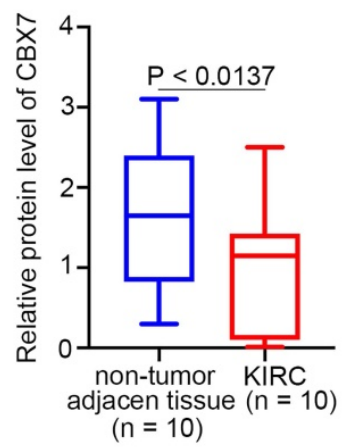

NAT
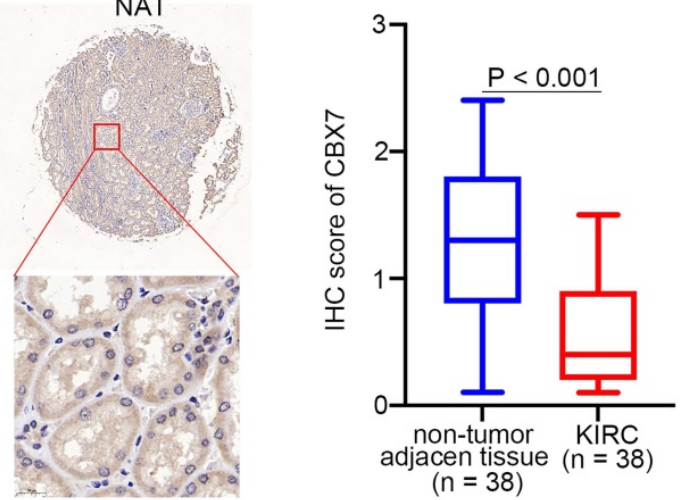

$(n=38)$

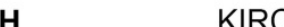

H KIRC

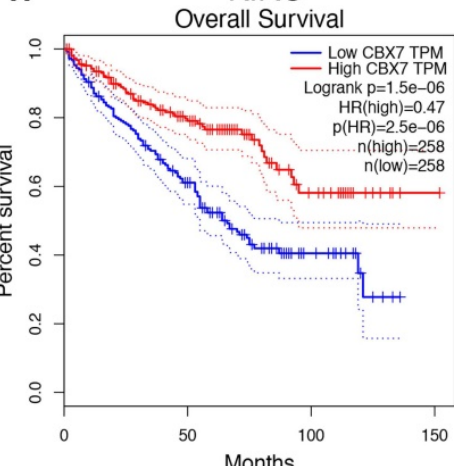

Figure 1. CBX7 is a protective factor in renal cell carcinoma. A, Univariate Cox regression analysis and multivariate Cox regression analysis adjusted for CBX1-8 in ccRCC. B, 10-fold cross-validation with 1000 replications for variable selection in the LASSO-COX-OS model by minimum criteria (the 1 -SE criteria). C, Differential expression analyses of RNF26 between tumor and normal tissues in TCGA-KIRC dataset. D, The protein expression levels of CBX7 in the adjacent non-tumor renal tissues ( $\mathrm{n}=10$ ) and ccRCC cancer tissues $(n=10)$ were analyzed by the western blot. The protein levels of CBX7 were quantified by the image J software. $P$ values as indicated in panel $D$. E, IHC analysis of the tissue microarray by staining the CBX7 antibody. The typical image and expression level of CBX7 in the non-tumor renal tissue and renal cancer tissue were shown. $P$ values as indicated. F, The CBX7 related overall survival in ccRCC analyzed by the Human protein atlas. $\mathbf{G}$ and $\mathbf{H}$, The CBX7 related disease free survival and overall survival in ccRCC analyzed by the Human protein atlas. 

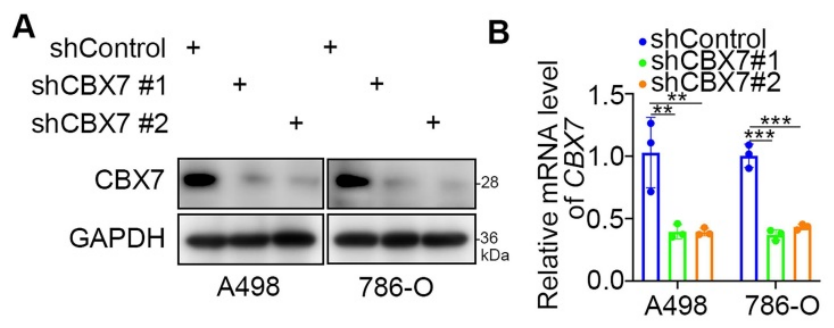

C

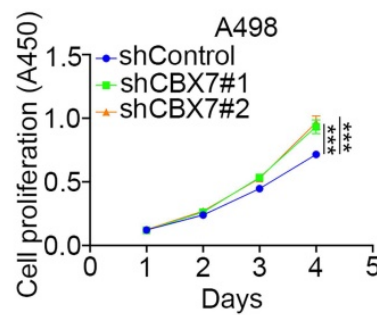

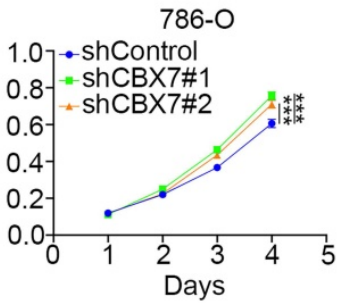

D

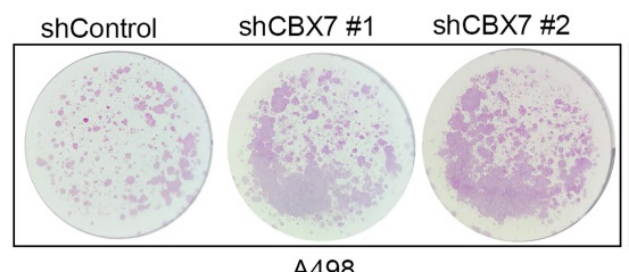

shControl shCBX7 \# shCBX7 \#2
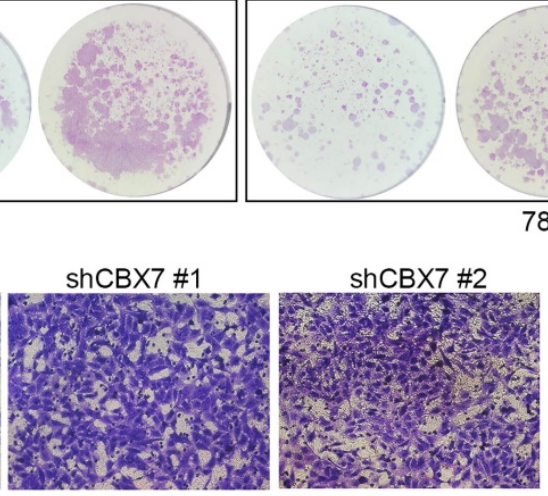

78-O

E

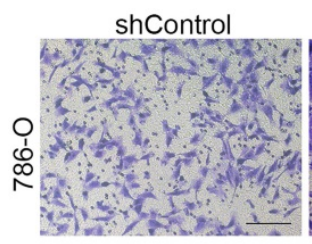

$\mathbf{F}$

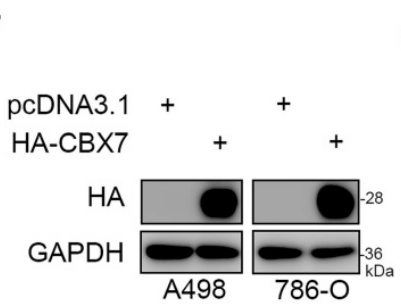

G

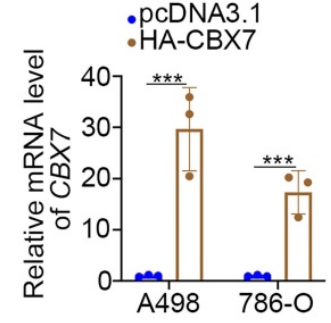

H
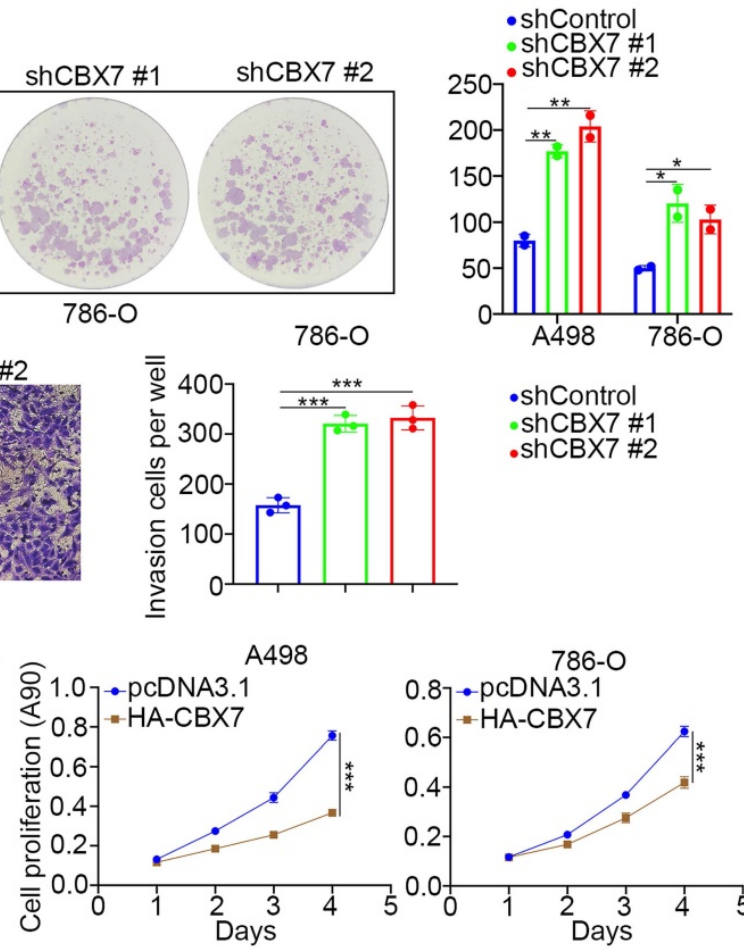

-shControl

- shCBX7 \#1

-shCBX7 \#2
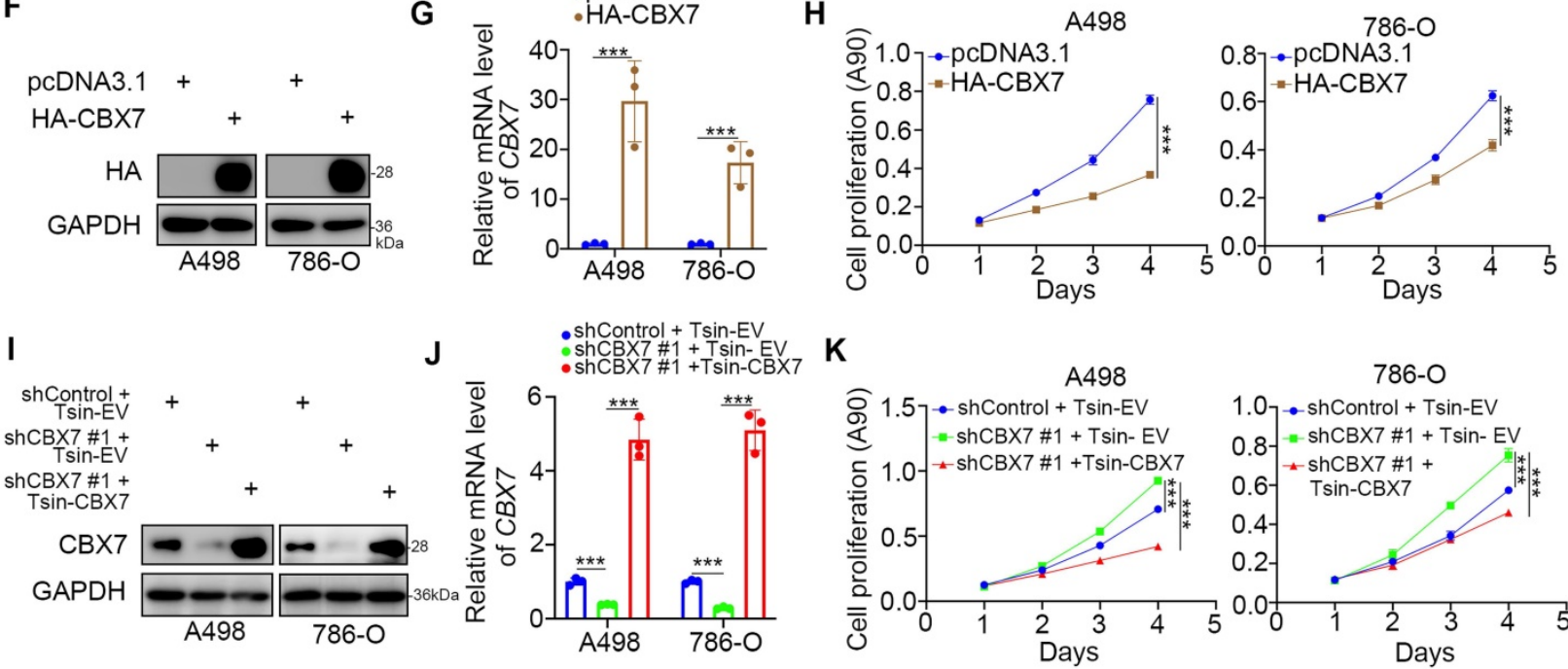

$786-0$ 
A

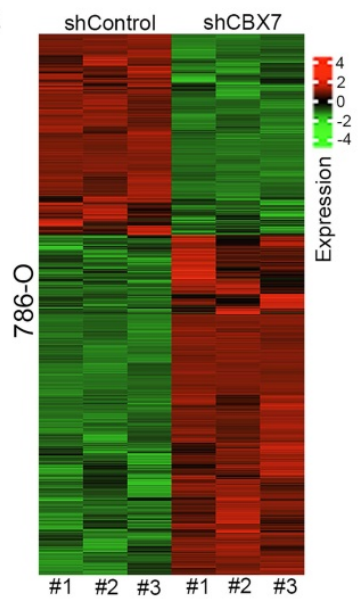

C

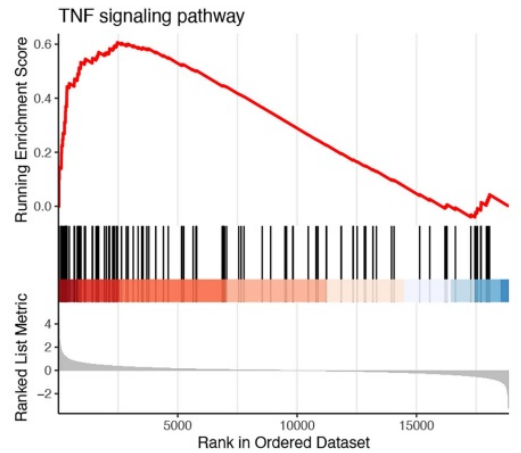

B

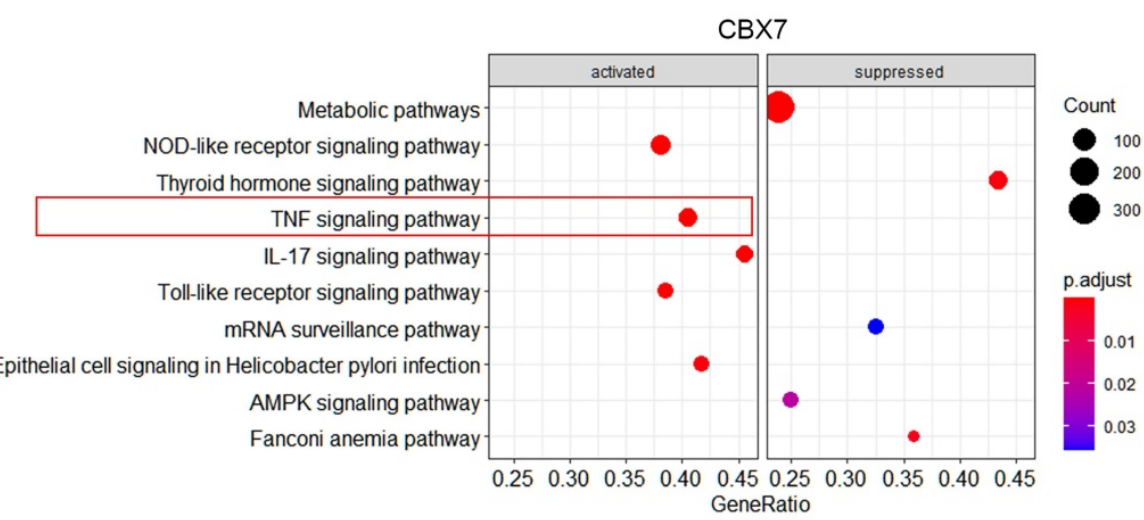

D

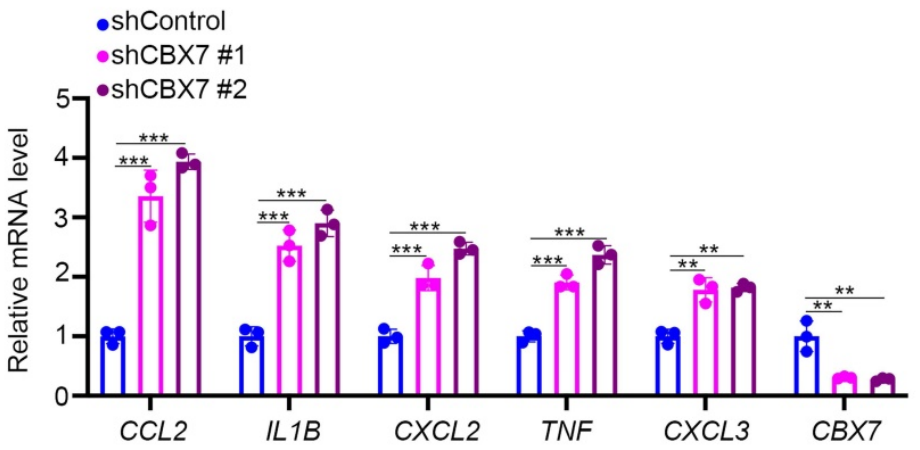

E

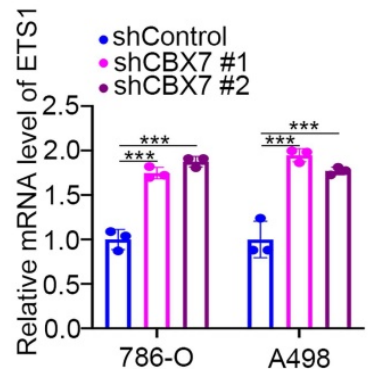

H

- SiNC

- SiETS1 \#1

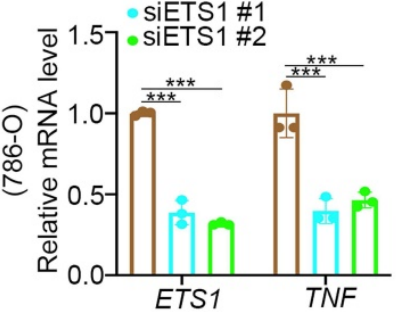

$\mathbf{F}$

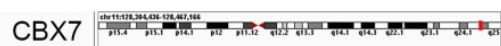
ChIP-seq ‥m,

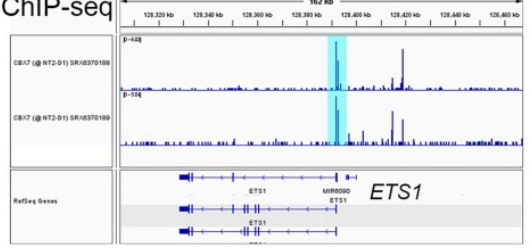

I

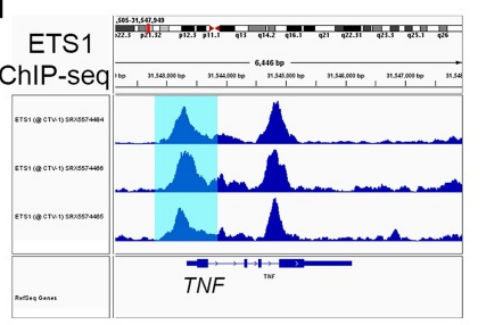

L

G
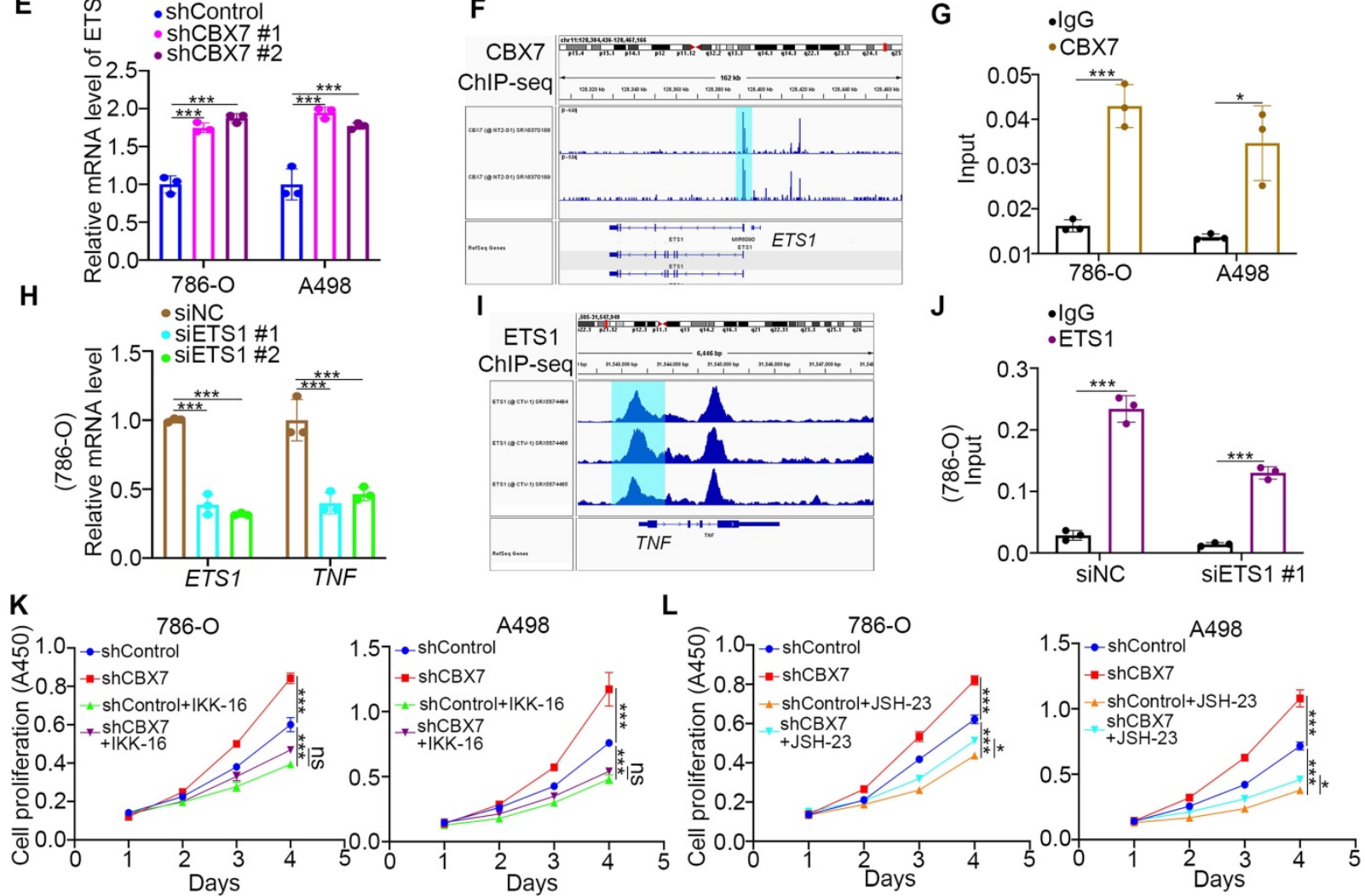

Figure 3. CBX7 blocks CcRCC tumorigenesis by inhibiting the TNF pathway. A, 786-O cells were infected with shControl or shCBX7 for $72 \mathrm{~h}$. Cells were subjected to RNA-seq analysis. B KEGG pathway enrichment analysis indicated that the pathways were changed after knockdown of CBX7 in 786-O cells. C, GSEA analysis showed TNF pathway was activated by knocking down CBX7 in 786-O cells. D, 786-O cells were infected with shControl or shCBX7 for $72 \mathrm{~h}$. Cells were collected for RT-qPCR analysis. Data presents as mean \pm SD with 3 replicates. $* *, P<0.01$; ***, $P<0.001$. E, $786-O$ and A498 cells were infected with shControl or shCBX7 for 72 h. Cells were collected for RT-qPCR analysis. Data presents as mean \pm SD with 3 replicates. ***, P $<0.001$. F, the ChIP-seq of CBX7 showed the binding peak of CBX7 in the promoter region of ETS1. G, 
786-O and A498 cells were subjected to ChIP-qPCR analysis by using the IgG and CBX7 antibodies. H, 786-O and A498 cells were transfected with siNC or siETSI for $48 \mathrm{~h}$. Data presents as mean \pm SD with 3 replicates. ***, $P<0.001$. I, the ChIP-seq of ETS1 showed the binding peak of ETS1 in the promoter region of TNF. J, 786-O cells were transfected with siNC or siETS1 for $48 \mathrm{~h}$. Cells were harvested for ChIP-qPCR analysis by using the $\operatorname{lgG}$ and ETS1 antibodies. K, 786-O and A498 cells were infected with shControl

As TNF is the stimulator of the TNF pathway, we performed ChIP-seq of CBX7 to determine whether CBX7 directly regulates the expression of TNF (Supplementary Fig. 2A). However, there was no binding peak of $\mathrm{CBX7}$ in the promoter region of TNF (Supplementary Fig. 2A). Thus, we intended to find a mediator of CBX7-induced TNF downregulation in ccRCC. By combining the RNA-seq data and ChIP-seq dataset, we identified five candidates, including ETS proto-oncogene 1 (ETS1), serum response factor (SRF), lysine demethylase 2B (KDM2B), cyclin dependent kinase 8 (CDK8) and core-binding factor subunit beta (CBFB) (Supplementary Fig. 2B). ETS1 was the most changed candidate after CBX7 silencing, and this phenomenon was observed in renal cancer cells (Fig. 3E, Supplementary Fig. 2C). We also showed that overexpression of CBX7 decreased ETS1 expression in renal cancer cells (Supplementary Fig. 2D). Additionally, ChIP-seq and subsequent ChIP-qPCR assays showed that CBX7 directly bound to the promoter region of ETS1 (Fig. 3F, G). Then, we demonstrated that knockdown of ETS1 decreased TNF expression and that ETS1 directly bound to the promoter region of TNF in 786-O cells (Fig. 3H-J). Since the TNF signaling pathway is responsible for the regulation of tumor growth via activating NF-kappa B pathway in renal cancer and other types of malignant tumor [11, 12], we tested whether CBX7 inhibited tumor proliferation through inactivation of the TNF pathway. We found that TNF pathway inhibitors treatment (IKK-16 and JSH-23) diminished the tumor growth-promoting effect induced by CBX7 knockdown in both 786-O and A498 cells (Fig. 3K, L).

\section{CBX7 contributes to regulating sensitivity to TKIs in cCRCC}

Tyrosine kinase inhibitors (TKIs) are the first-line therapy for ccRCC. Of note, gene set enrichment analysis (GSEA) of the RNA-seq data for CBX7 indicated that $\mathrm{CBX7}$ might be involved in EGFR tyrosine kinase inhibitor resistance (Fig. 4A), and the levels of a series of genes related to modulating sensitivity to TKIs were found to be changed after CBX7 silencing (Fig. 4B). The subsequent cellular study showed that knockdown of CBX7 increased the IC50 values of sunitinib and pazopanib in both 786-O and A498 cells (Fig. 4C, D). In contrast, overexpression of $\mathrm{CBX7}$ reduced the IC50 values of sunitinib and pazopanib in renal cancer cells (Fig. 4E, F). We found that interleukin 6 (IL6) was upregulated after CBX7 silencing, as shown in Figure 4B, and IL6 is a well-known factor responsible for the resistance of renal cancer cells to TKIs. It was not surprising that IL6 neutralizing antibodies attenuated the CBX7-induced sensitivity change of TKIs (Fig. 4G, H). Then, we explored the mechanism by which CBX7 regulates the expression of IL6 in renal cancer cells. Although knockdown/overexpression of CBX7 increased/decreased IL6 expression in ccRCC cells (Fig. 4I, J,Supplementary Fig. 2C,2D), ChIP-seq of CBX7 demonstrated that CBX7 did not directly bind to the promoter region of IL6 (Supplementary Fig. 2E). IL6 is the downstream gene of TNF signaling [13], and CBX7 contributes to the repression of TNF signaling in renal cancer cells (Figure 3). We showed that inhibitors of TNF signaling diminished the increase in IL6 after CBX7 knockdown in 786-O cells (Fig. 4K, L). These results showed that CBX7 regulated IL6 expression through TNF signaling pathway. Thus, we revealed that $\mathrm{CBX7}$ regulated sensitivity to TKIs through the TNF/IL6 axis in ccRCC.

\section{RNF26 interacts with CBX7 and decreases CBX7 expression in CCRCC}

Since downregulation of CBX7 is important for the progression of $\mathrm{CCRCC}$, the regulatory mechanism of CBX7 in ccRCC is worthy of further study. Regulation of protein stability by ubiquitination is a major aspect of posttranslational modification. Protein-protein interaction (PPI) network analysis indicated that the E3 ligase RNF26 was closely associated with CBX7 in renal cancer (Fig. 5A, B). The coimmunoprecipitation assay showed that exogenously expressed CBX7 bound to RNF26 in 293T cells (Fig. 5C). Then, we found that endogenously expressed CBX7 interacted with RNF26 in both 786-O and A498 cells (Fig. 5D, 5E). Consistent with this result, the GST pulldown assay demonstrated that there was an interaction between RNF26 and CBX7 in vitro (Fig. 5F). We further evaluated the relationship between RNF26 and CBX7 in renal cancer cells after knocking down RNF26. We found that RNF26 silencing increased the protein levels of $\mathrm{CBX7}$ and had no effect on the mRNA levels of CBX7 in renal cancer cells (Fig. 5G, H). Moreover, IHC staining of a renal cancer tissue microarray with anti-CBX7 and anti-RNF26 antibodies demonstrated that CBX7 expression was negatively correlated with RNF26 expression in the patient specimens (Spearman correlation $r=-0.349, \mathrm{P}=0.0318$ ) (Fig. 5I, J). Therefore, our results indicate that RNF26 is a binding partner of $\mathrm{CBX7}$ and is involved in decreasing the protein level of CBX7 in RCC. 
A

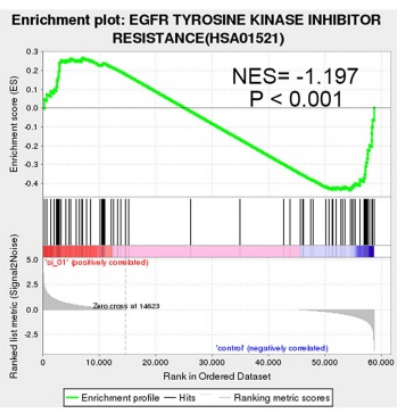

C

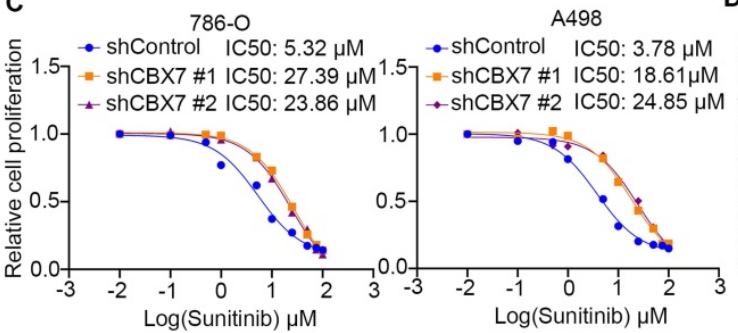

E $786-0$

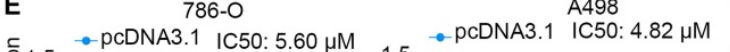

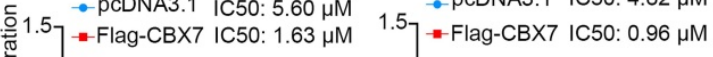

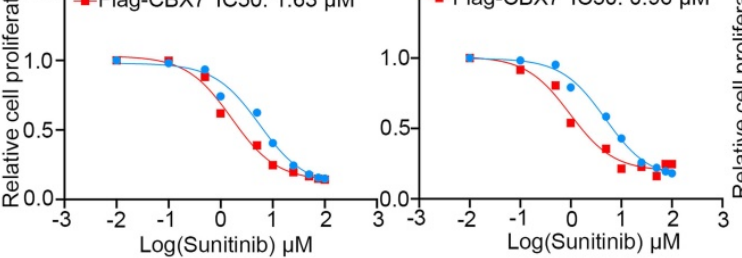

G

786-0
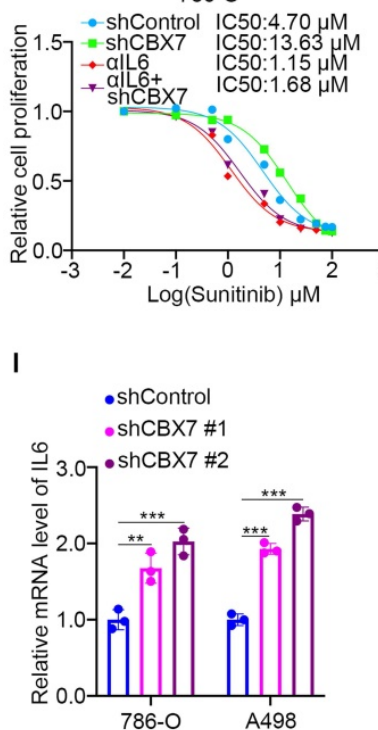

B

D

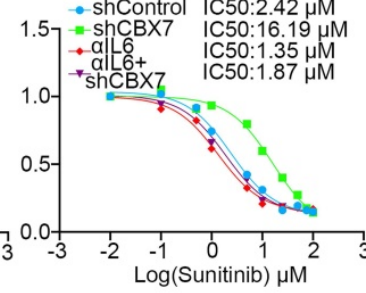

J

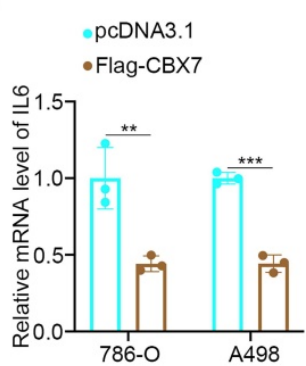

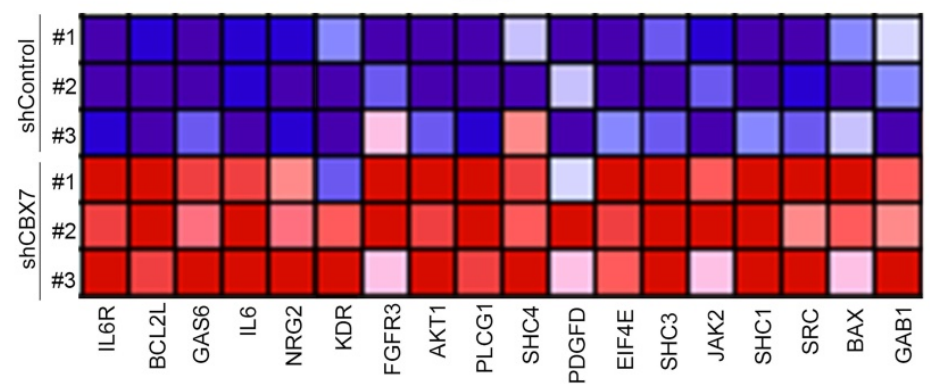

786-0

A498

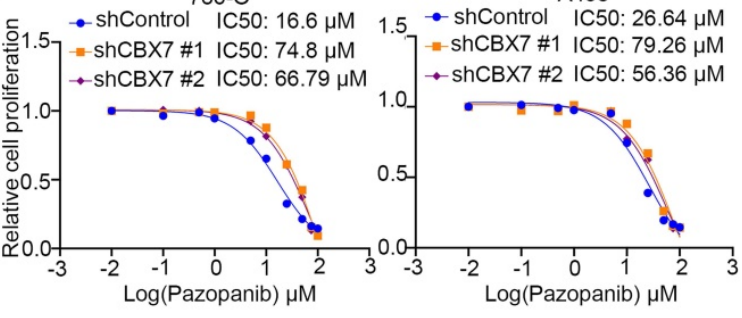

F $786-0$

A498

$786-0$
pcDNA3.1 IC50: $16.74 \mu \mathrm{M}$

을 1.57 -Flag-CBX7 IC50: $\left.5.75 \mu \mathrm{M} \quad{ }^{1.5}\right]=$ Flag-CBX7 IC50: $2.48 \mu \mathrm{M}$

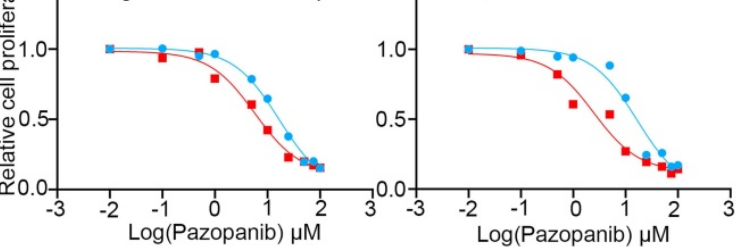

H

H - shControl IC50:2.42 $\mu \mathrm{M}$

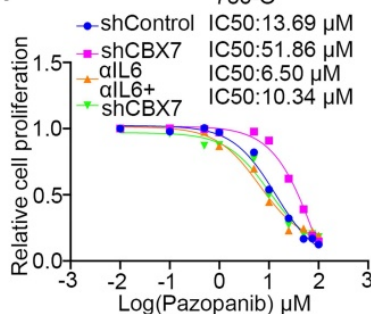

A498 $1.5=$ shCBX7 IC50:64.18 $\mu \mathrm{M}$ alL6 IC50:5.42 $\mu \mathrm{M}$

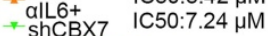

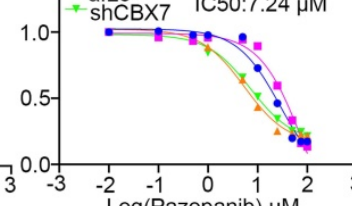

K

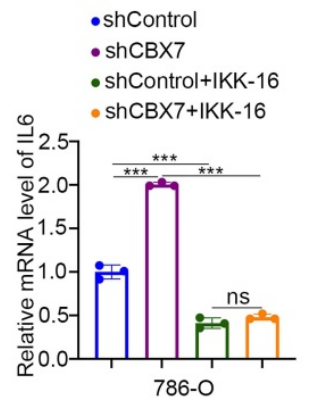

L

- shControl

- shControl+JSH-23

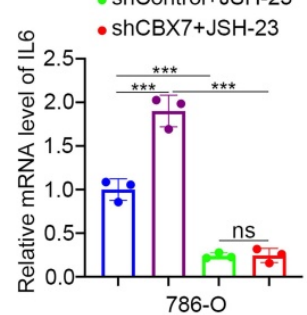

Figure 4. CBX7 contributes to regulating sensitivity to TKIs in ccRCC. A, GSEA analysis showed EGFR tyrosine kinase inhibitors resistance related pathway was activated by knocking down CBX7 in 786-O cells. B, the genes involved EGFR tyrosine kinase inhibitors resistance related pathway after knocking down CBX7 in 786-O cells. C, 786-O and A498 cells were infected with indicated shRNAs for $72 \mathrm{~h}$. Cells were treated with a serial concentration of Sunitinib and harvested for CCK-8 assay. D, 786-O and A498 cells were infected with indicated shRNAs for $72 \mathrm{~h}$. Cells were treated with a serial concentration of Pazopanib and harvested for CCK-8 assay. E, 786-O and A498 cells were transfected with indicated constructs for $24 \mathrm{~h}$. Cells were treated with a serial concentration of Sunitinib and harvested for CCK-8 assay. F, 786-O and A498 cells were transfected with indicated constructs for $24 \mathrm{~h}$. Cells were treated with a serial concentration of Pazopanib and harvested for CCK-8 assay. G, 786-O and A498 cells were infected with indicated shRNAs for $72 \mathrm{~h}$. Cells were pretreated with or without IL6 for $6 \mathrm{~h}$. Then, these cells were treated with a serial concentration of Sunitinib and harvested for CCK-8 assay. H, 786-O and A498 cells were transfected with indicated constructs for $24 \mathrm{~h}$. Cells were pretreated with or without IL6 for $6 \mathrm{~h}$. Then, these cells were treated with a serial concentration of Sunitinib and harvested for CCK-8 assay. I, 786-O and A498 cells were infected with indicated shRNAs for $72 \mathrm{~h}$. Cells were harvested for RT-qPCR analysis. Data presents as mean \pm SD with 3 replicates. **, $\mathrm{P}<0.01$; ***, $\mathrm{P}<0.001$. J, 786-O and A498 cells were transfected with indicated plasmids for 24 h. Cells were harvested for RT-qPCR analysis. Data presents as mean \pm SD with 3 replicates. $* *, P<0.01$; ***, $P<0.001$. K, $786-O$ cells were infected with indicated shRNAs for 72 h. Cells were treated with or without IKK-16 for another $24 \mathrm{~h}$. Cells were harvested for RT-qPCR analysis. Data presents as mean \pm SD with 3 replicates. ***, P $<0.001$. L, 786-O cells were infected with indicated shRNAs for $72 \mathrm{~h}$. Cells were treated with or without JSH-23 for another $24 \mathrm{~h}$. Cells were harvested for RT-qPCR analysis. Data presents as mean \pm SD with 3 replicates. ${ }^{* * *}, \mathrm{P}<0.001$. 
A

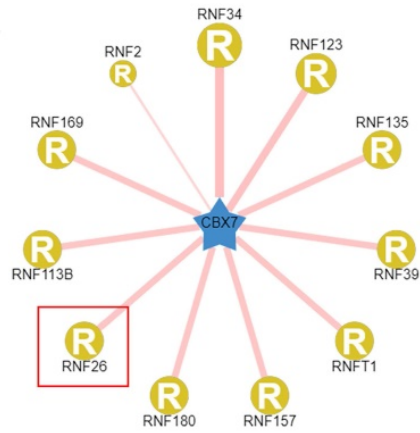

B

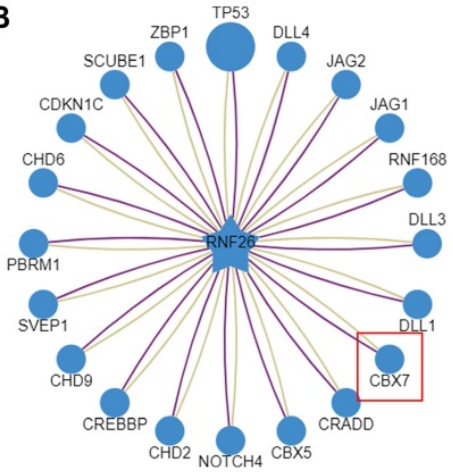

$\mathbf{F}$

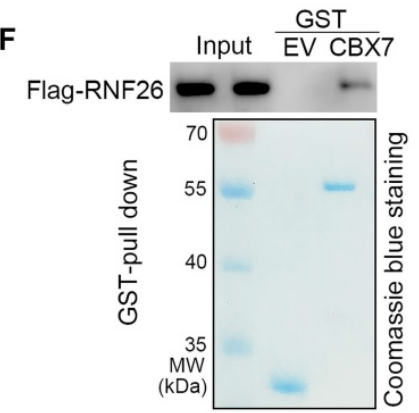

I

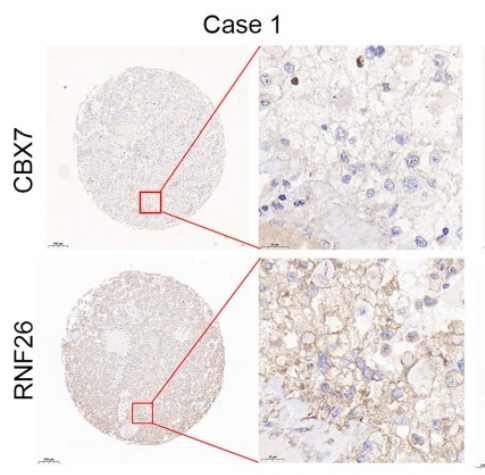

G

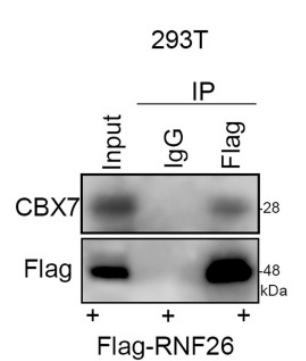

D

786-0

E

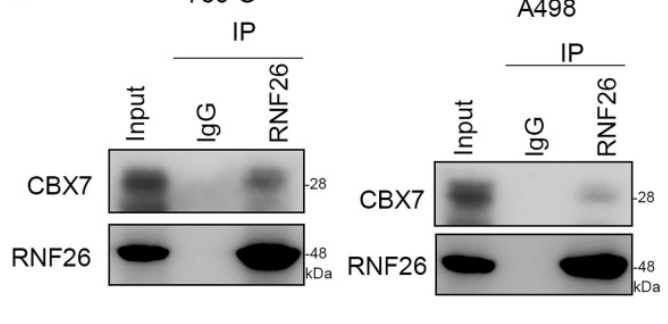

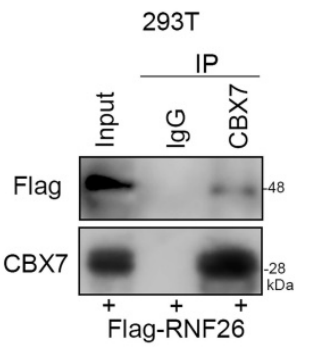
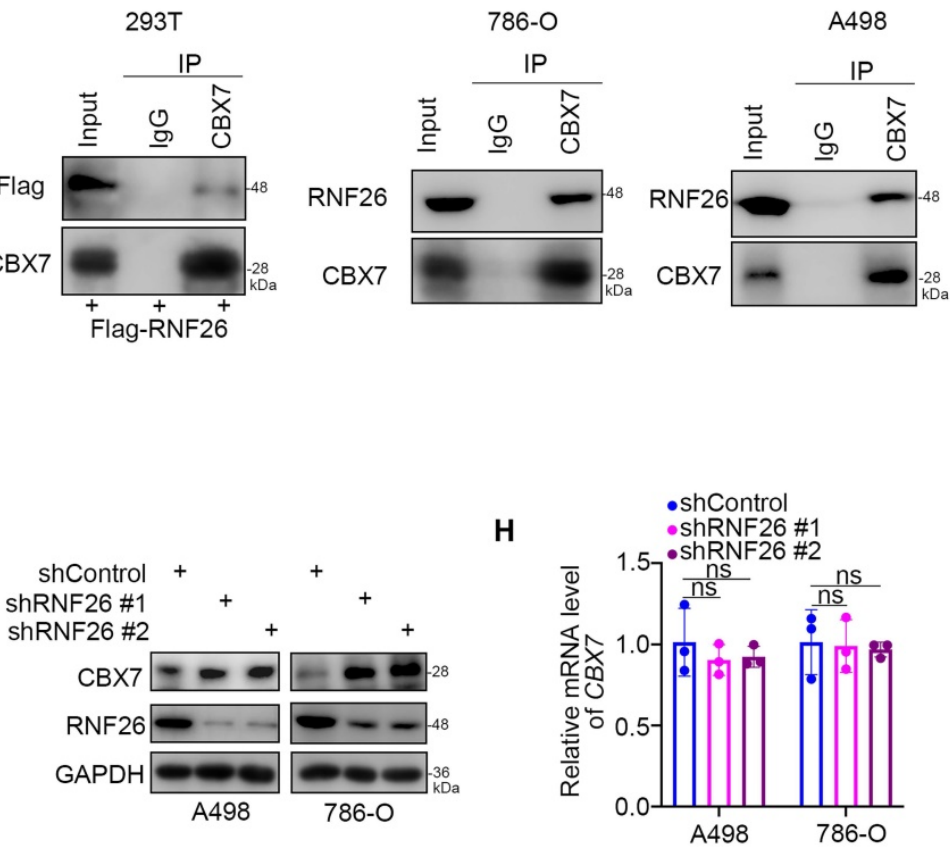

$J$

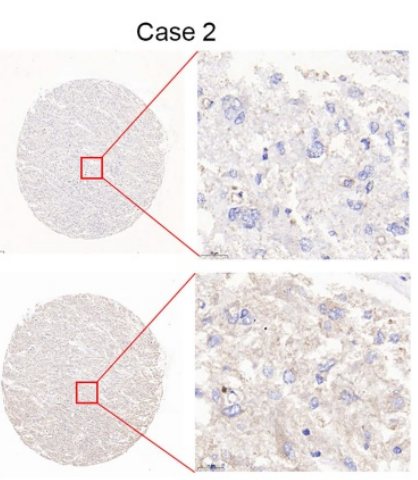

Spearman $r=-0.349, P=0.0318$

$\mathrm{n}=38$

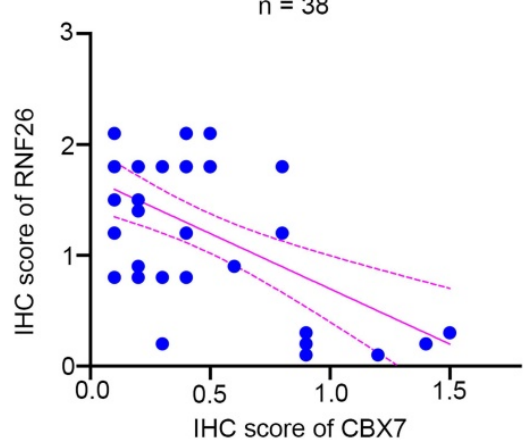

Figure 5. RNF26 interacts with CBX7 and decreases CBX7 expression in ccRCC. A, UbiBrowser showed substrate proteins that might interact with CBX7. B, UbiBrowser showed substrate proteins that might interact with RNF26. C, 293T cells were transfected with indicated plasmids. Cells were collected and immunoprecipitated with IgG and CBX7 or Flag antibodies. D and E, 786-O and A498 cells were harvested and immunoprecipitated with IgG and CBX7 or RNF26 antibodies. F, Western blot analysis for Flag-RNF26 in 786-O cells after GST or GST-CBX7 pulldown. The bottom panel shows the Coomassie blue staining of GST or GST-CBX7 protein input. G and H, A498 and 786-O cells were infected with indicated shRNAs for $72 \mathrm{~h}$. Cells were harvested for Western blot (G) and RT-qPCR analysis. Data presents as mean \pm SD with 3 replicates. Ns, not significant. I and J, Renal cancer tissue microarray was stained for CBX7 and RNF26. Representative images are shown in panel I. The correlation of CBX7 and RNF26 levels is shown in panel J; P-values are also shown in the figure.

\section{RNF26 promotes the degradation of CBX7 in renal cancer cells}

To further explore the relationship between RNF26 and CBX7 in renal cancer cells, we treated renal cancer cells with or without a $26 \mathrm{~S}$ proteasome inhibitor (MG132) under conditions of knockdown or overexpression of RNF26 (Fig. 6A, B). We showed that MG132 effectively blocked the upregulation or downregulation of CBX7 induced by knockdown or 
overexpression of RNF26 in A498 and 786-O cells (Fig. $6 \mathrm{~A}, \mathrm{~B})$. Moreover, we showed that overexpression of wild-type (WT) RNF26 but not the functionally dead mutant (C401S) reduced the protein level of CBX7 in A498 and 786-O cells [14] (Fig. 6C). In addition, we found that knockdown of RNF26 prolonged the protein half-life of CBX7 and deceased the
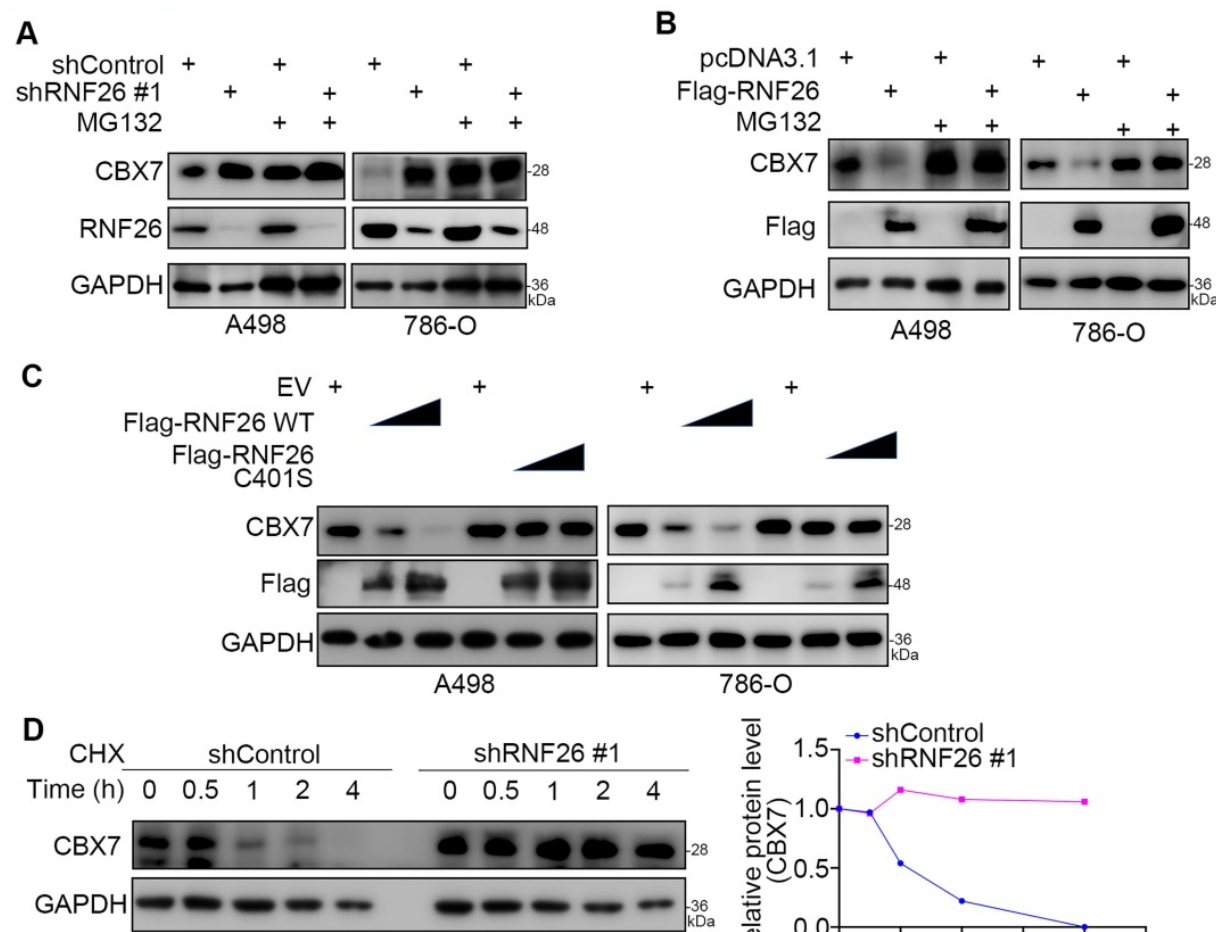

polyubiquitination level of CBX7 in 786-O cells (Fig. $6 \mathrm{~d}, \mathrm{~g})$. However, overexpression of RNF26 WT but not the C401S mutant shortened the protein half-life of CBX7 and increased the polyubiquitination level of CBX7 in 786-O cells (Fig. 6e, f). Thus, our data indicated that RNF26 regulated the protein stability of CBX7 in renal cancer cells.

B

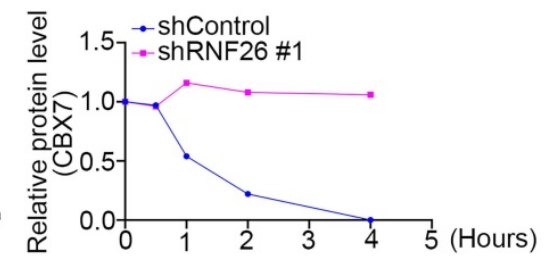

E
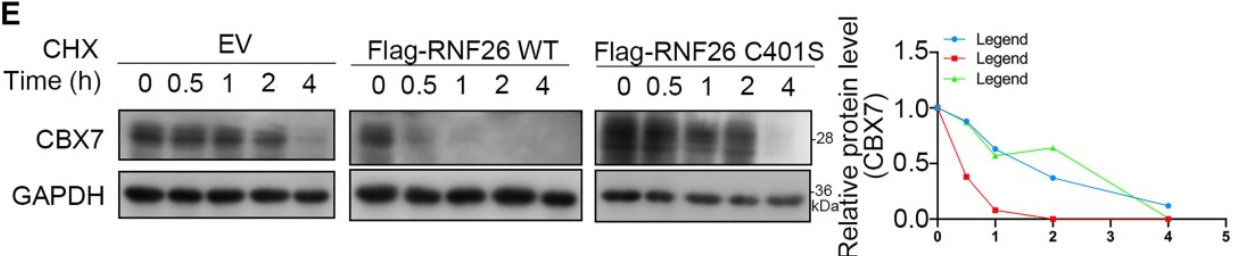

$\mathbf{F}$

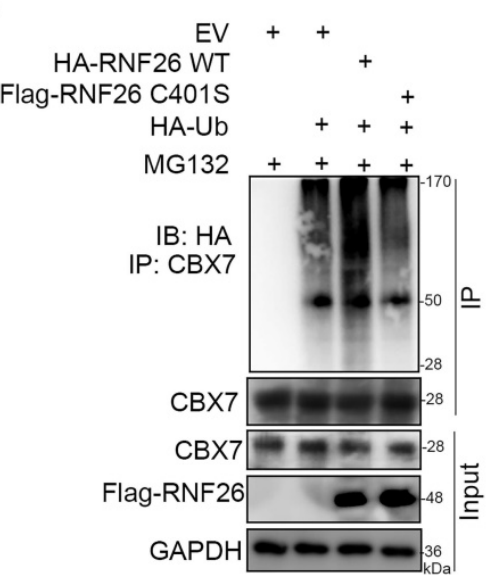

G

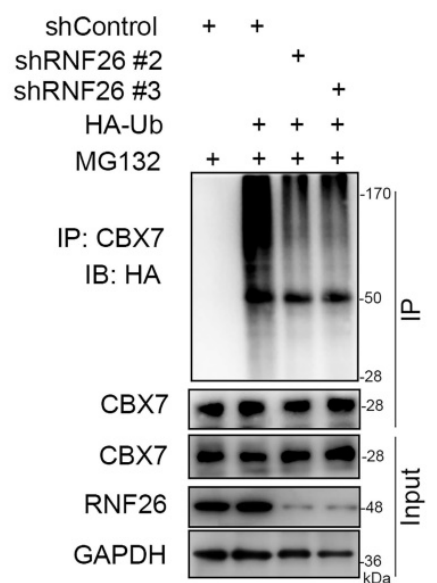

Figure 6. RNF26 promotes the degradation of CBX7 in renal cancer cells. A, A498 and 786-O cells were infected with indicated shRNAs for $72 \mathrm{~h}$. After treated with or without MG1 32 for another $8 \mathrm{~h}$, cells were harvested for Western blot analysis. B, A498 and 786-O cells were transfected with indicated plasmids for $24 \mathrm{~h}$. After treated with or without MG132 for another $8 \mathrm{~h}$, cells were harvested for Western blot analysis. C, A498 and 786-O cells were transfected with indicated plasmids for 24 h. Cells were harvested for Western blot analysis. D, 786-O cells were infected with the indicated shRNAs. After $72 \mathrm{~h}$, cells were treated with cycloheximide (CHX), and cells were collected for Western blot analysis at different time points. E, 786-O cells were transfected with the indicated plasmids. After $24 \mathrm{~h}$, cells were treated with $\mathrm{CHX}$, and cells were collected for Western blot analysis at different time points. F, 786-O cells were transfected with the indicated plasmids. After $24 \mathrm{~h}$, cells were collected for Western blot after treatment with MG132 for 8 h. G, 786-O cells were infected with the indicated shRNAs. After 72 h, cells were collected for Western blot after treatment with MG132 for 8 h. 


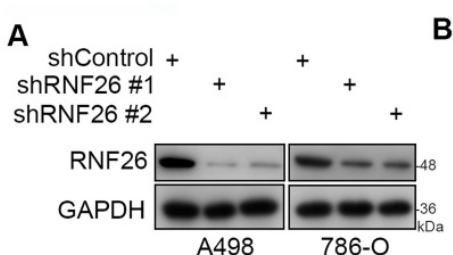

D

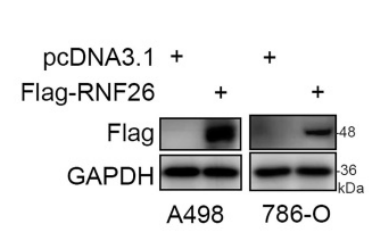

G

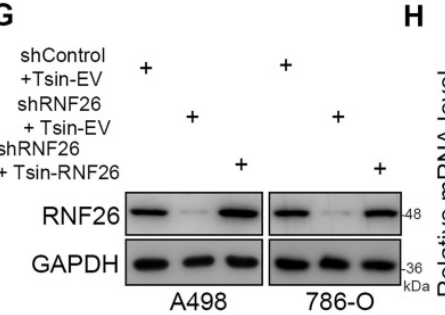

J

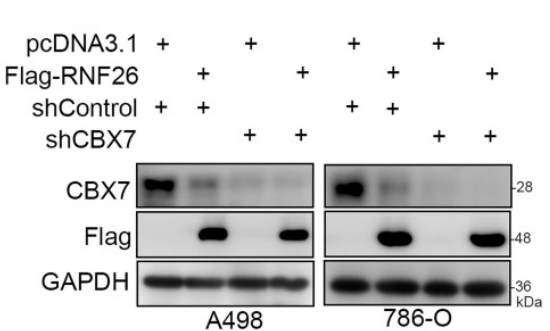

K

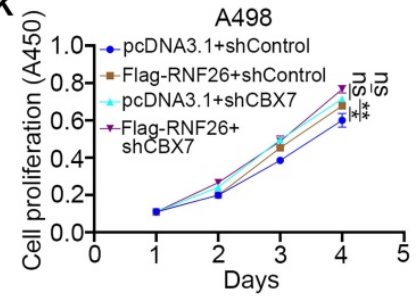

.pcDNA3.1
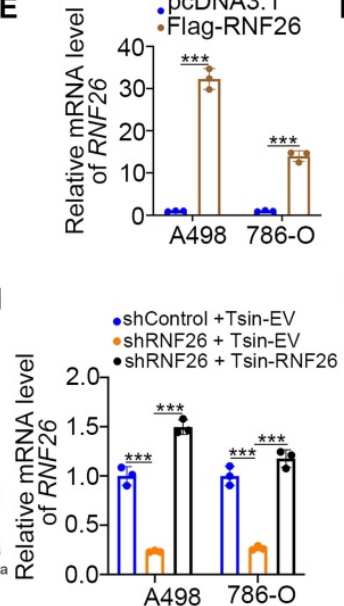

C
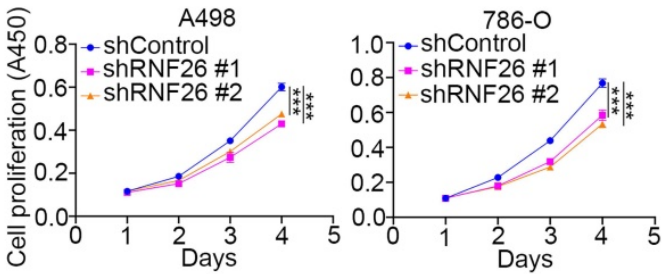

F O $A 498$

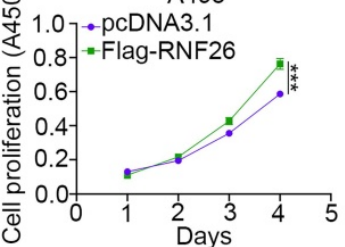

1
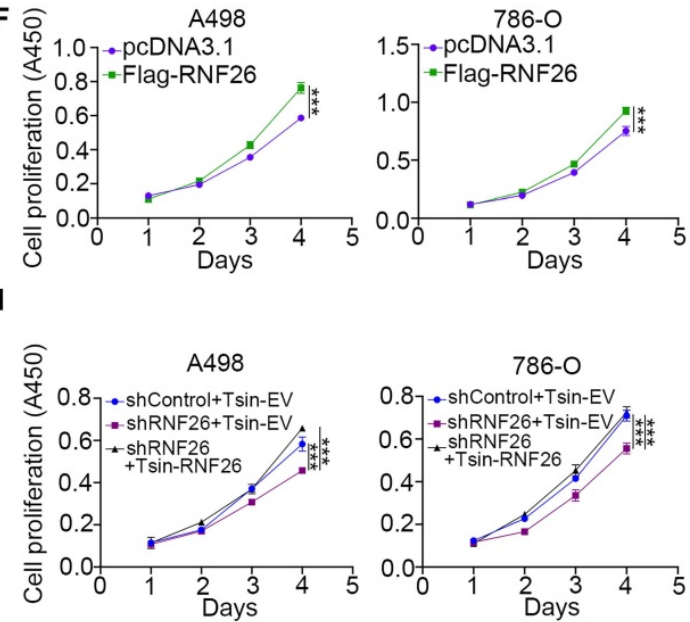

786-O

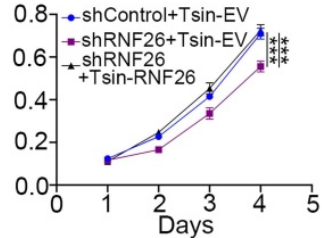

786-O

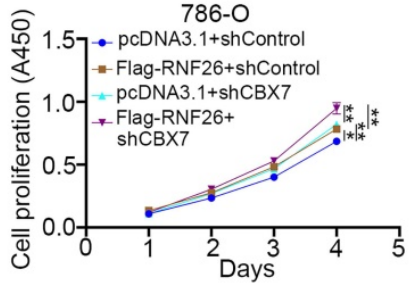

$\mathbf{L}$
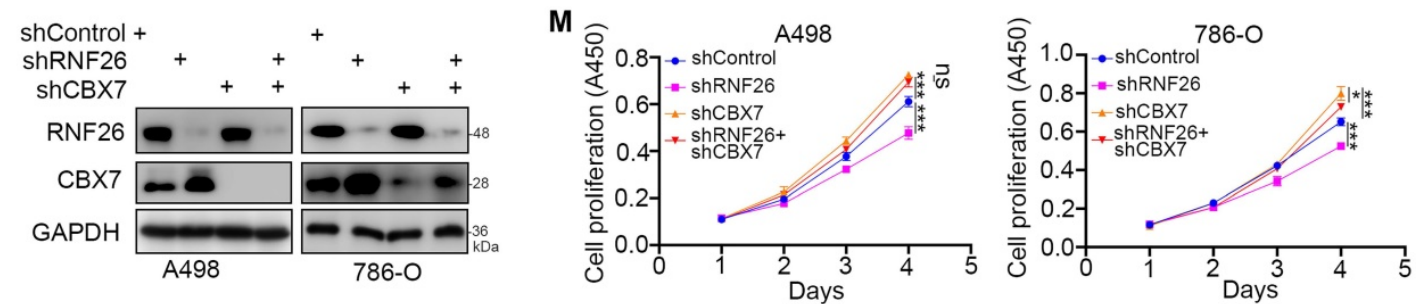

$\mathbf{N}$

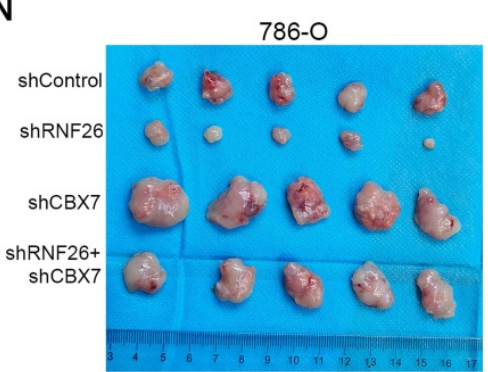

0

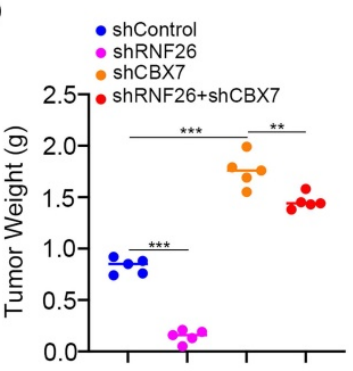

$\mathbf{P}$

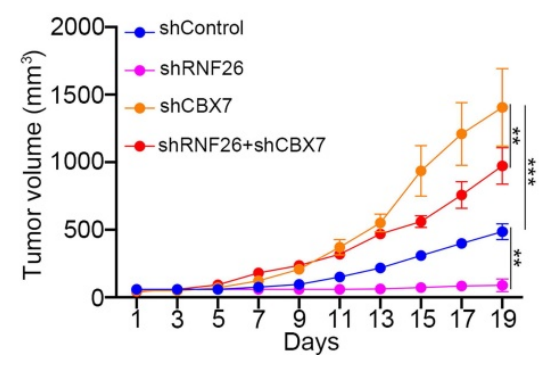

Figure 7. CBX7 is a key mediator of RNF26-induced RCC progression. A-C, A498 and 786-O cells were infected with indicated shRNAs for $72 \mathrm{~h}$. Cells were collected for Western blot analysis (A), RT-qPCR (B) and CCK-8 assay (C). Data presents as mean \pm SD with 3 replicates. ***, P<0.001. D-F, A498 and 786-O cells were transfected with indicated plasmids for $24 \mathrm{~h}$. Cells were collected for Western blot analysis (D), RT-qPCR (E) and CCK-8 assay (F). Data presents as mean \pm SD with 3 replicates. $* * *, P<0.001$. G-I, A498 and 786-O cells were infected with indicated constructs for $72 \mathrm{~h}$. Cells were collected for Western blot analysis (G), RT-qPCR (H) and CCK-8 assay (I). Data presents as mean \pm SD with 3 replicates. ***, P $<0.001$. J and $\mathrm{K}$, A498 and 786-O cells were infected with indicated constructs for $72 \mathrm{~h}$. Cells were collected for Western blot analysis $(J)$ and CCK-8 assay (K). Data presents as mean \pm SD with 3 replicates. Ns, not significant; *, P $<0.05 ; * *, P<0.01 ; * * *, P<0.001$. L and M, A498 and 786-O cells were infected with indicated shRNAs for $72 \mathrm{~h}$. Cells were collected for Western blot analysis (L) and CCK-8 assay (M). Data presents as mean \pm SD with 3 replicates. Ns, not significant; *, P $<0.05$; ***, $\mathrm{P}<0.001$. N-P, $786-\mathrm{O}$ cells were infected with indicated constructs for $72 \mathrm{~h}$. After puromycin selection, cells were subcutaneously injected into the nude mice. The image of xenografts was shown in the panel $\mathrm{N}$. The tumor mass was shown in the panel $\mathrm{O}$. The tumor growth curve was shown in the panel P. Data presents as mean \pm SD with 5 replicates. $* *, P<0.01 ; * * *, P<0.001$. 


\section{CBX7 is a key mediator of RNF26-induced RCC progression}

Next, we evaluated the cancer-related role of RNF26 in RCC. It was not surprising that knockdown of RNF26 decreased the proliferation ability of renal cancer cells (Fig. 7A-C). In contrast, overexpression of RNF26 promoted renal cancer cell growth (Fig. 7D-F). In addition, rescue of RNF26 expression reversed the growth-suppressive effect induced by RNF26 silencing (Fig. 7G-I). Notably, compared with overexpression of RNF26 alone, overexpression of RNF26 in CBX7 knockdown cells did not further increase cancer cell growth (Fig. 7J, K). In addition, we also showed that combined knockdown of CBX7 and RNF26 attenuated the tumor growth inhibition effect induced by knockdown of RNF26 alone in renal cancer cells and a subcutaneous xenograft model (Fig. 7L-P). Together, our data suggest that RNF26 might promote renal cell proliferation via CBX7.

\section{Discussion}

Studies have reported that different CBX family members perform distinct functions in cancer. Recently, Zhu, et al performed comprehensive analysis of the expression and prognosis value of CBX1-8 in ccRCC [15]. They demonstrated that CBX3 and CBX4 were up-regulated in the ccRCC tissues and associated with the poor prognosis, but CBX1, CBX5, $\mathrm{CBX} 6$, and $\mathrm{CBX7}$ were down-regulated in the cCRCC tissues and correlated with the favorable prognosis [15]. In this study, we performed bioinformatic analysis to show that CBX7 and CBX6 functioned as protective factors with hazard ratios (HRs) less than 1 but CBX8 and CBX4 functioned as risk factors with HRs greater than 1 in renal cell carcinoma (Fig. 1A). The clinicopathological roles of CBX4 and CBX7 were consistent in these two studies with opposite roles in ccRCC, respectively. Here, we focused on studying the cancer related role of CBX7 in ccRCC. CBX7 expression is lost in a variety of cancers, such as urothelial carcinoma [16], colorectal cancer [17], breast cancer [18], pancreatic cancer [19] and lung cancer [20]. In urothelial carcinoma, the expression of CBX7 showed a close correlation with high tumor grade [16]. In addition, a clear correlation between downregulation of $\mathrm{CBX7}$ and poor prognosis has been found in many types of cancers [21]. Consistent with previous findings, our data indicated that the CBX7 level was decreased in ccRCC and was a protective factor for ccRCC prognosis. In addition, the tumor-associated role of CBX7 has been studied. It has been reported that $\mathrm{CBX7}$ hinders HDAC2 activity to promote $\mathrm{CDH} 1$ expression and block epithelial-mesenchymal transition in thyroid, pancreatic and cervical cancer cells [19, 22-24]. In addition, CBX7 has been found to suppress pancreatic cancer progression by inhibiting AKT signaling [25] and to inhibit tumorigenicity in breast cancer through the Wnt/ $\beta$-catenin pathway [26]. However, CBX7 also acts as an oncogenic protein in gastric cancer by upregulating p16 [27]. Thus, the role of CBX7 in cancer is controversial. Here, we demonstrated that CBX7 inactivated the TNF signaling pathway through downregulation of ETS1 expression in ccRCC cells, which was reported to transcriptionally increase the expression of TNF [28]. Several pathways contribute to the progression of ccRCC, such as Hypoxia signaling pathway [29] and PI3K-AKT signaling pathway [30]. Of them, TNF alpha mediated NF-kappa B signaling activation also plays a key role in promoting the tumor growth in cCRCC [11]. It has been reported that TNF alpha induced eotaxin-1 expression to enhance the progression of renal cancer cells through CCR3 [11]. Recently, Du et al have demonstrated that activation of the NF-kappa B pathway increased the ccRCC tumor growth capability through upregulating stathmin [31]. Meanwhile, the absence of von Hippel Lindau gene (VHL) gene enhances the activity of NF-kappa B, which subsequently leads to drug resistance and epithelial-mesenchymal-transition of RCC [32]. Here, we showed that CBX7 blocked tumor proliferation through inactivation of the TNF pathway and regulated sensitivity to TKIs via the TNFa/IL6 axis. While, the TNFa/NF-kappa B signaling was not the only pathway for CBX7 to inhibit the tumor growth in cCRCC, the underlying mechanism by which CBX7 modulates the cancer progression needs to be performed in the furture.

CBX7 loss is a common feature in many cancers, including renal cancer. The underlying mechanism needs in-depth study. HMGA1 has been found to directly bind to the promoter of CBX7 and transcriptionally repress CBX7 expression [18]. In addition, several microRNAs, such as miR-421, miR-181b, miR182 and miR-183, have been reported to suppress CBX7 expression [21, 33]. Separate from the transcriptional regulation of $\mathrm{CBX7}$, we focused on the posttranscriptional modification of $\mathrm{CBX7}$ in renal cancers. We demonstrated that RNF26 interacted with CBX7 and promoted CBX7 degradation in renal cancer cells, which provides novel insight into the low expression of CBX7 in cancers. Of note, the PPI network analysis suggested that CBX7 might bind with other E3 ligases. Thus, the regulatory mechanism of CBX7 degradation needs further exploration. 


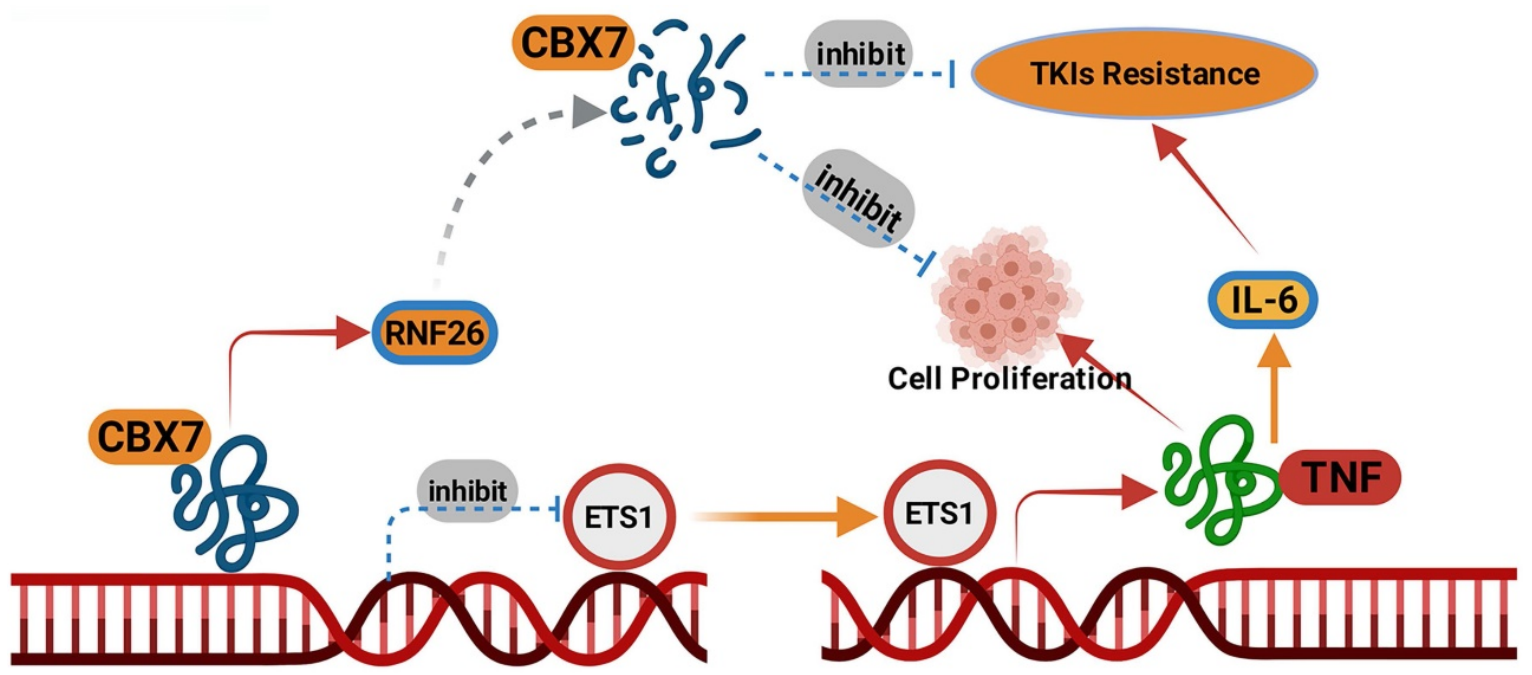

Figure 8. A model depicting that CBX7was degraded by RNF26. The downregulated CBX7 inhibited the ETS1 expression and subsequently inactivated TNF pathway to block the tumor growth and overcome the TKIs resistance in ccRCC.

RNF26 belongs to the RING finger protein with a C-terminal RING finger domain [34]. RNF26 promotes K11-linked polyubiquitination of MITA and degradation of IRF3 to trigger type I interferon [14]. Meanwhile, the endoplasmic reticulum (ER)-located RNF26 induces the ubiquitination of the scaffold p62/sequestosome 1 and regulates of the endocytic and exocytic systems [35]. The dysregulation of RNF26 in cancer is rarely reported. We previously showed that RNF26 was abnormally upregulated in bladder cancer and promoted the tumor growth through destabilizing p57 [36]. Here, we demonstrated that CBX7 was another substrate of RNF26 for degradation in renal cancer cells. And RNF26 degraded CBX7 to promote the renal cancer cells proliferation. We believed that more substrates of RNF26 will be identified in the further to explain the role of RNF26 in malignant tumor.

Collectively, our results demonstrated that CBX7 was downregulated in ccRCC tissues and associated with favorable prognosis in patients with ccRCC. Then, we showed that CBX7 inactivated the TNF signaling pathway to inhibit tumor proliferation and enhanced the sensitivity of ccRCC cells to TKIs. Moreover, we found that CBX7 was a bona fide substrate of RNF26. RNF26 promoted the degradation of CBX7 and enhanced ccRCC tumor growth (Fig. 8). Therefore, our results revealed a novel RNF26/CBX7 axis that modulates the TNF signaling pathway in ccRCC.

\section{Abbreviations}

TCGA: The Cancer Genome Atlas; ANOVA: one-way analysis of variance; KIRC: Kidney Renal Clear Cell Carcinoma; GEPIA: Gene Expression Profiling Interactive Analysis; RNF26: ring finger protein 26; TKI: tyrosine kinase inhibitors; CBX7: Chromobox protein homolog 7; ChIP: Chromatin immunoprecipitation; IHC: immunohistochemistry; ccRCC: Clear cell renal cell carcinoma; ETS1: ETS proto-oncogene 1; SRF: serum response factor; KDM2B: lysine demethylase 2B; CDK8: cyclin dependent kinase 8; CBFB: core-binding factor subunit beta; RNF: ring finger protein; TNF: tumor necrosis factor; HDAC1: histone deacetylase 1; KLF6: Kruppel like factor 6; CCL2: C-C motif chemokine ligand 2; IL1B: interleukin 1 beta; CXCL2: C-X-C motif chemokine ligand 2; IL6: interleukin 6.

\section{Supplementary Material}

Supplementary materials and methods, figures and tables. https://www.ijbs.com/v18p2132s1.pdf

\section{Acknowledgements}

\section{Funding}

This work was supported by grants from the Hunan Provincial Development and Reform Commission (Grant No.2019875, 2021319 (L.Y.)); Hunan Provincial Health Commission (Grant No. 20200816 (L.Y.)); Department of Science and Technology of Hunan Province (Grant No. 2020SK2082 (L.Y.)).

\section{Ethics approval and consent to participate}

The study was conducted in accordance with the principles of the Declaration of Helsinki principles. It was approved by the Animal Use and Care Committees at the Second Xiangya hospital, Central South University (Approval number: 2021613).

\section{Author Contributions}

$\mathrm{Lu}$ Yi: Funding acquisition, Project 
administration; Xin Jin: Investigation, Methodology, Project administration, Writing - Original Draft; Wentao Liu: Methodology, Software; Haohui Wang: Methodology; Chengzhu Jian: Methodology; Wei Li: Methodology; Kun Ye: Investigation; Jiannan Ren: Conceptualization; Liang Zhu: Software, Formal analysis; Yinhuai Wang: Project administration.

\section{Consent for publication}

All subjects have written informed consent.

\section{Availability of data and material}

The datasets used and/or analyzed during the current study are available from the corresponding authors (Xin Jin, jinxinxy2@csu.edu.cn) on reasonable request.

\section{Competing Interests}

The authors have declared that no competing interest exists.

\section{References}

1. Hsieh JJ, Purdue MP, Signoretti S, Swanton C, Albiges L, Schmidinger M, et al. Renal cell carcinoma. Nat Rev Dis Primers. 2017; 3: 17009.

2. Linehan WM, Ricketts CJ. The Cancer Genome Atlas of renal cell carcinoma: findings and clinical implications. Nat Rev Urol. 2019; 16: 539-52.

3. Roskoski R, Jr. Vascular endothelial growth factor (VEGF) and VEGF receptor inhibitors in the treatment of renal cell carcinomas. Pharmacol Res. 2017; 120: 116-32.

4. Makhov P, Joshi S, Ghatalia P, Kutikov A, Uzzo RG, Kolenko VM. Resistance to Systemic Therapies in Clear Cell Renal Cell Carcinoma: Mechanisms and Management Strategies. Mol Cancer Ther. 2018; 17: 1355-64.

5. Ma RG, Zhang Y, Sun TT, Cheng B. Epigenetic regulation by polycomb group complexes: focus on roles of CBX proteins. J Zhejiang Univ Sci B. 2014; 15: 412-28

6. Plys AJ, Davis CP, Kim J, Rizki G, Keenen MM, Marr SK, et al. Phase separation of Polycomb-repressive complex 1 is governed by a charged disordered region of CBX2. Genes Dev. 2019; 33: 799-813.

7. Liang YK, Lin HY, Chen CF, Zeng D. Prognostic values of distinct CBX family members in breast cancer. Oncotarget. 2017; 8: 92375-87.

8. Jiang $\mathrm{N}$, Niu G, Pan $\mathrm{YH}$, Pan $\mathrm{W}$, Zhang MF, Zhang $\mathrm{CZ}$, et al. CBX4 transcriptionally suppresses KLF6 via interaction with HDAC1 to exert oncogenic activities in clear cell renal cell carcinoma. EBioMedicine. 2020; 53: 102692.

9. He H, Yi L, Zhang B, Yan B, Xiao M, Ren J, et al. USP24-GSDMB complex promotes bladder cancer proliferation via activation of the STAT3 pathway. Int J Biol Sci. 2021; 17: 2417-29.

10. Jin X, Ding D, Yan Y, Li H, Wang B, Ma L, et al. Phosphorylated RB Promotes Cancer Immunity by Inhibiting NF-kappaB Activation and PD-L1 Expression. Mol Cell. 2019; 73: 22-35 e6.

11. Johrer K, Zelle-Rieser C, Perathoner A, Moser P, Hager M, Ramoner R, et al. Up-regulation of functional chemokine receptor CCR3 in human renal cell carcinoma. Clin Cancer Res. 2005; 11: 2459-65.

12. Chen L, Zhou J, Li L, Zhao J, Li H, Zheng W, et al. SLC39A7 promotes malignant behaviors in glioma via the TNF-alpha-mediated NF-kappaB signaling pathway. J Cancer. 2021; 12: 4530-41.

13. Lee KM, Jeon SM, Cho HJ. Tumor necrosis factor receptor 1 induces interleukin-6 upregulation through NF-kappaB in a rat neuropathic pain model. Eur J Pain. 2009; 13: 794-806.

14. Qin Y, Zhou MT, Hu MM, Hu YH, Zhang J, Guo L, et al. RNF26 temporally regulates virus-triggered type $\mathrm{I}$ interferon induction by two distinct mechanisms. PLoS Pathog. 2014; 10: e1004358.

15. Zhu Y, Pu Z, Li Z, Lin Y, Li N, Peng F. Comprehensive Analysis of the Expression and Prognosis Value of Chromobox Family Members in Clear Cell Renal Cell Carcinoma. Front Oncol. 2021; 11: 700528.

16. Hinz S, Kempkensteffen C, Christoph F, Krause H, Schrader M, Schostak M, et al. Expression parameters of the polycomb group proteins BMI1, SUZ12, RING1 and CBX7 in urothelial carcinoma of the bladder and their prognostic relevance. Tumour Biol. 2008; 29: 323-9.

17. Pallante P, Terracciano L, Carafa V, Schneider S, Zlobec I, Lugli A, et al. The loss of the CBX7 gene expression represents an adverse prognostic marker for survival of colon carcinoma patients. Eur J Cancer. 2010; 46: 2304-13.
18. Mansueto $G$, Forzati F, Ferraro A, Pallante P, Bianco M, Esposito F, et al. Identification of a New Pathway for Tumor Progression: MicroRNA-181b Up-Regulation and CBX7 Down-Regulation by HMGA1 Protein. Genes Cancer. 2010; 1: 210-24.

19. Karamitopoulou E, Pallante P, Zlobec I, Tornillo L, Carafa V, Schaffner T, et al. Loss of the CBX7 protein expression correlates with a more aggressive phenotype in pancreatic cancer. Eur J Cancer. 2010; 46: 1438-44.

20. Forzati F, Federico A, Pallante P, Abbate A, Esposito F, Malapelle U, et al. CBX7 is a tumor suppressor in mice and humans. J Clin Invest. 2012; 122: 612-23.

21. Pallante P, Forzati F, Federico A, Arra C, Fusco A. Polycomb protein family member CBX7 plays a critical role in cancer progression. Am J Cancer Res. 2015; 5: 1594-601.

22. Federico A, Pallante P, Bianco M, Ferraro A, Esposito F, Monti M, et al. Chromobox protein homologue 7 protein, with decreased expression in human carcinomas, positively regulates E-cadherin expression by interacting with the histone deacetylase 2 protein. Cancer Res. 2009; 69: 7079-87.

23. Thiery JP. Epithelial-mesenchymal transitions in tumour progression. Nat Rev Cancer. 2002; 2: 442-54.

24. Li R, Yan Q, Tian P, Wang Y, Wang J, Tao N, et al. CBX7 Inhibits Cell Growth and Motility and Induces Apoptosis in Cervical Cancer Cells. Mol Ther Oncolytics. 2019; 15: 108-16.

25. Ni S, Wang H, Zhu X, Wan C, Xu J, Lu C, et al. CBX7 suppresses cell proliferation, migration, and invasion through the inhibition of PTEN/Akt signaling in pancreatic cancer. Oncotarget. 2017; 8: 8010-21.

26. Kim HY, Park JH, Won HY, Lee JY, Kong G. CBX7 inhibits breast tumorigenicity through DKK-1-mediated suppression of the Wnt/beta-catenin pathway. FASEB J. 2015; 29: 300-13.

27. Zhang XW, Zhang L, Qin W, Yao XH, Zheng LZ, Liu X, et al. Oncogenic role of the chromobox protein CBX7 in gastric cancer. J Exp Clin Cancer Res. 2010; 29: 114 .

28. Zhang XM, Guo L, Huang X, Li QM, Chi MH. 4-Hydroxynonenal Regulates TNF-alpha Gene Transcription Indirectly via ETS1 and microRNA-29b in Human Adipocytes Induced From Adipose Tissue-Derived Stromal Cells. Anat Rec (Hoboken). 2016; 299: 1145-52.

29. Baik AH. Hypoxia signaling and oxygen metabolism in cardio-oncology. J Mol Cell Cardiol. 2022; 165: 64-75.

30. Aurilio G, Santoni M, Massari F, Cimadamore A, Rizzo A, Mollica V, et al. Metabolomic Profiling in Renal Cell Carcinoma Patients: News and Views. Cancers (Basel). 2021; 13.

31. Shi D, Zhang Z, Kong C. CARMA3 Transcriptional Regulation of STMN1 by NF-kappaB Promotes Renal Cell Carcinoma Proliferation and Invasion. Technol Cancer Res Treat. 2021; 20: 15330338211027915

32. Morais C, Gobe G, Johnson DW, Healy H. The emerging role of nuclear factor kappa B in renal cell carcinoma. Int J Biochem Cell Biol. 2011; 43: 1537-49.

33. Pallante P, Battista S, Pierantoni GM, Fusco A. Deregulation of microRNA expression in thyroid neoplasias. Nat Rev Endocrinol. 2014; 10: 88-101.

34. Katoh M. Molecular cloning and characterization of RNF26 on human chromosome 11q23 region, encoding a novel RING finger protein with leucine zipper. Biochem Biophys Res Commun. 2001; 282: 1038-44.

35. Jongsma ML, Berlin I, Wijdeven RH, Janssen L, Janssen GM, Garstka MA, et al. An ER-Associated Pathway Defines Endosomal Architecture for Controlled Cargo Transport. Cell. 2016; 166: 152-66.

36. Yi L, Wang H, Li W, Ye K, Xiong W, Yu H, et al. The FOXM1/RNF26/p57 axis regulates the cell cycle to promote the aggressiveness of bladder cancer. Cell Death Dis. 2021; 12: 944. 